\title{
A Fully Supersymmetric AKNS Theory
}

\author{
Carlo Morosi ${ }^{1}$, Livio Pizzocchero ${ }^{2}$ \\ ${ }^{1}$ Dipartimento di Matematica, Politecnico di Milano, Piazza L. da Vinci 32, I-20133 Milano, Italy \\ 2 Dipartimento di Matematica, Università di Milano, Via C. Saldini 50, I-20133 Milano, Italy and \\ Istituto Nazionale di Fisica Nucleare, Sezione di Milano, Italy. \\ E-mail: PIZZOCCHERO@VMIMAT.MAT.UNIMI.IT
}

Received: 18 April 1994/Accepted: 15 July 1995

\begin{abstract}
We construct a fully supersymmetric biHamiltonian theory in four superfields, admitting zero curvature and Lax formulation. This theory is an extension of the classical AKNS, which can be recovered as a reduction. Other supersymmetric theories are obtained as reductions of the susy AKNS, namely a nonlinear Schrödinger, a modified KdV and the Manin-Radul KdV. The susy nonlinear Schrödinger hierarchy is related to the one of Roelofs and Kersten; we determine its biHamiltonian and Lax formulation. Finally, we show that the susy KdV's mentioned before are related through a susy Miura map.
\end{abstract}

\section{Introduction and Preliminaries}

In the last decade there has been increasing interest in superextensions of the soliton evolution equations. The earlier results concerned the construction of field theories with fermionic and bosonic fields depending on time and one space variable $x$ [Kup]. Next, the susy (=supersymmetric) soliton equations were investigated. In the so-called $N=1$ susy extensions [MR], in which we are mainly interested, the field variables depend, apart from time, on the superspace variables $x, \theta$, with $x$ even and $\theta$ odd; the field equations are formulated in terms of the superderivative $D=\theta \partial / \partial x+\partial / \partial \theta$, with the property $D^{2}=\partial / \partial x$. Also, the $N=2$ susy extensions were introduced, with one even and two odd superspace variables $x, \theta_{1}, \theta_{2}$ [Mat]; in this case, two superderivatives $D_{i}=\theta_{i} \partial / \partial x+\partial / \partial \theta_{i}$ are employed.

The best known methods for constructing soliton equations can be appropriately generalized to the susy framework. The Lax formalism in terms of fractional powers was extended by introducing an algebra of pseudodifferential operators in $D$ (or $D_{1}$ and $D_{2}$ ); in this way, some susy $\mathrm{KdV}$ equations were constructed [MR, Mat, LM], and the corresponding biHamiltonian structures were obtained via $R$-matrix theory [OP, FMR].

Moreover, the connections between the susy soliton equations and the theory of Lie superalgebras were analyzed in [IK1-4, MP1], in order to obtain a superanalogue of the classical Drinfeld-Sokolov theory [DS]. 
Recently, the method of prolongation structures [EW] was extended to field theories with fermionic variables $[\mathrm{RH}]$; as a byproduct, a super nonlinear Schrödinger (NLS) hierarchy was obtained by Roelofs and Kersten [RK], involving a bosonic field $q=q(x)$ and a fermionic field $\omega=\omega(x)$. The vector fields and the conservation laws of this hierarchy were written in terms of $q, \omega$ and their $x$-derivatives; moreover, it was found that the super NLS equation admits an $N=1$ susy formulation in terms of the superfield $\psi(x, \theta):=\omega(x)+\theta q(x)$ and its superderivatives. (This super NLS equation is different from the one proposed previously in [Kul, CN], for which a susy formulation in the above sense was not given).

In our previous works [MP1-4], we discussed the susy $\mathrm{KdV}$ equations from a biHamiltonian and Lie superalgebraic viewpoint. Following this approach, in this paper we propose an $N=1$ susy extension of the AKNS theory, involving two even superfields $b=b(x, \theta), a=a(x, \theta)$ and two odd superfields $\psi=\psi(x, \theta)$, $\varphi=\varphi(x, \theta)$. This theory consists of a hierarchy of commuting vector fields, possessing infinitely many conservation laws; we explicitly construct the biHamiltonian formulation, giving a pair of compatible Poisson structures for which all the vector fields of the hierarchy are Hamiltonian. Moreover, we give the zero curvature representation and the Lax formulation. The classical AKNS hierarchy in two field variables $q=q(x), r=r(x)$ can be recovered through the reduction

$$
b=a=0, \quad \psi(x, \theta)=\theta q(x), \quad \varphi(x, \theta)=\theta r(x) .
$$

Both the biHamiltonian structure and the spectral problem of the susy AKNS can be reduced to the classical ones via Eq. (1.1).

On the other side, if we put the constraints

$$
b=a=0, \quad \varphi(x, \theta)=\overline{\psi(x, \theta)},
$$

we get a susy extension of the ordinary NLS theory; by inspection of the first vector fields and conservation laws, it is found that they can be converted into the homologous objects of the Roelofs-Kersten super NLS hierarchy. The advantages of our approach are:

i) the intrinsically supersymmetric formulation of the hierarchy, which is constructed directly in terms of the superfield $\psi(x, \theta)$ and its superderivatives;

ii) the fact that we give a biHamiltonian and a Lax formalism.

We also consider two alternative reductions of the susy AKNS, which are defined by the constraints

$$
a=b, \quad \varphi=\psi \quad \text { and } \quad b=-1, \quad \psi=0,
$$

respectively. The first reduction gives rise to a modified susy $\mathrm{KdV}$ theory; the second one generates the susy KdV of Manin and Radul [MR]. By comparing the associated Lax formulations, we obtain a susy Miura map relating the two theories. (As is known, the classical AKNS theory contains the NLS, modified KdV and KdV theories as reductions; so, the susy extension considered in this paper possesses the counterparts of these classical features).

We now illustrate the plan of the paper and the theoretical setting from which the results came about.

In Sect. 2, we present the main results about the susy AKNS, giving the first vector fields and conservation laws, the biHamiltonian structure (Table 1a) and the zero curvature/Lax formulation (Table 1b). The reductions mentioned above are discussed in Sect. 3 and the main results are summarized in Tables 2-4. 
Subsequently, we illustrate the method we have employed to generate the susy AKNS; in Sect. 4, we consider in particular the biHamiltonian structure and in Sect. 5 we derive the zero curvature/Lax formalism. Most of the computations in the paper have been carried out using the MATHEMATICA symbolic manipulation system; in order to save space, only the essential part of the computational results has been reported in the tables.

As for the theoretical setting, we refer to the methods discussed in general in the previous papers [MP1-2]; different applications of these techniques were also presented in [MP3-4]. As anticipated above, our approach is essentially Lie superalgebraic and biHamiltonian; it is based on a geometrical viewpoint which was widely developed for the classical soliton equations (see, in particular, [FF, MMR, DS, CMP, CP]).

The main geometrical objects in our setting are the loop superalgebras, i.e. Lie superalgebras of maps $V=V(x, \theta)$, taking values in some finite-dimensional Lie superalgebra. The odd part $\mathscr{G}_{1}$ of a loop superalgebra $\mathscr{G}$ is a biHamiltonian manifold, i.e., it carries two compatible Poisson tensors $Q$ and $P$; at any point $V$ we have

$$
Q_{V}(\delta V)=[A, \delta V], \quad P_{V}(\delta V)=D(\delta V)+[V, \delta V]
$$

for each covector $\delta V$ (identified with an element in the even part $\mathscr{G}_{0}$ of the loop superalgebra). In the above equation, $A$ denotes a fixed element of $\mathscr{G}_{1}$, whose choice is in principle arbitrary. The susy AKNS theory discussed in this paper is derived working with the loop superalgebra $\mathscr{G}=\operatorname{gl}(2,2)\{x, \theta\}$; the elements of $\mathscr{G}$ are $4 \times 4$ matrices, the entries being scalar superfields. $A$ is the (constant) matrix $\left(\begin{array}{cccc}0 & 0 & 0 & 0 \\ 0 & 0 & 0 & 0 \\ -1 & 0 & 0 & 0 \\ 0 & 1 & 0 & 0\end{array}\right)$.

The biHamiltonian structure (1.4) can be reduced using a technique proposed recently in [CMP, CP], where the Marsden-Ratiu reduction theorem for general Poisson manifolds [MaR] was specialized to the biHamiltonian case. In these papers, it was shown that the Poisson tensors of a general biHamiltonian manifold can be reduced to a conveniently defined quotient manifold, and this result was applied to loop algebras in connection with the classical $\mathrm{KdV}$-type equations. The method also works in a susy framework [MP1]; in our previous papers, it was applied to the loop superalgebra $\mathscr{G}=g l(2,2)\{x, \theta\}$, giving rise on the quotient space to the biHamiltonian structure of the Manin-Radul susy KdV.

Here, we are working with the same loop algebra, but the matrix $A$ defining the first Poisson tensor in Eq. (1.4) is different from the one considered in previous works. The choice of $A$ related to the Manin-Radul theory was made on the grounds of a natural susy extension of the Drinfeld-Sokolov "lowest root criterion" [DS], also considered in [IK2]; here, we explore a different possibility, which is suggested by the analogy with the classical AKNS case. With this new choice, the quotient manifold of the reduction turns out to be a space of quadruples $(b, a, \psi, \varphi)$, where the two first components are even superfields $b=b(x, \theta), a=a(x, \theta)$, and the other two are odd superfields $\psi=\psi(x, \theta), \varphi=\varphi(x, \theta)$. Using the reduced biHamiltonian structure, we generate with a standard procedure a hierarchy of vector fields and Hamiltonian functions: this is the susy AKNS.

The next steps in our construction are the zero curvature and the Lax formalism of the hierarchy, which are natural outcomes of the biHamiltonian reduction [MP2]. The zero curvature representation can be seen as a Lax formulation with 
parameter, the Lax operator being a $4 \times 4$ matrix first order differential operator in the superderivative $D$. From here, it is possible to derive a reduced, parameter independent Lax formalism, where the Lax operator is a $2 \times 2$ matrix differential operator acting on a space of pairs $\left(\begin{array}{l}\Psi_{1} \\ \Psi_{2}\end{array}\right)$, each component being a superfield $\Psi_{i}=\Psi_{i}(x, \theta)$. The eigenvalue equation for this operator is

$$
\left\{\begin{array}{l}
-\partial_{x} \Psi_{1}-\psi D \Psi_{2}-b \Psi_{2}=\lambda \Psi_{1} \\
\partial_{x} \Psi_{2}+\varphi D \Psi_{1}+a \Psi_{1}=\lambda \Psi_{2}
\end{array}\right.
$$

which appears to be a natural susy extension of the classical AKNS spectral problem.

The biHamiltonian and Lax formalism of the susy AKNS can be carried over to the reductions mentioned before, i.e., the susy NLS, modified KdV and Manin-Radul $\mathrm{KdV}$. In the case of the two KdV's, it is also possible to derive in a geometrical fashion the scalar Lax formulation in terms of fractional powers.

This is, summing up, the content of the paper. Before going into the details of the susy AKNS, we add some (very short and informal) preliminaries about the basic supermathematics employed in the paper; this is useful for uniformity of language. Also, we recall the definition of the Lie superalgebra $g l(2,2)$ and the associated loop algebra.

1.1. Linear superalgebra. We extensively refer to the framework of the previous work [MP1]. The "numbers" or "scalars" we employ are the elements of a super $\left(=\mathbb{Z}_{2}\right.$-graded $)$ algebra. More precisely, we have a real associative superalgebra with unit $L=L_{0} \oplus L_{1}$, whose elements commute in the graded sense (i.e., elements in the even part $L_{0}$ commute with every element of $L$, and elements in the odd part $L_{1}$ mutually anticommute). The complexification of $L$ is the complex superalgebra $\Lambda=$ $\Lambda_{0} \oplus \Lambda_{1}$, where, for $k=0,1, \Lambda_{k}:=\left\{u+i v \mid u, v \in L_{k}\right\}$; we define complex conjugation setting $\overline{u+i v}:=u-i v$ for each $u, v \in L$. The whole $L$ (or its even part $L_{0}$ ) plays a role similar to the real numbers, while $\Lambda$ (or $\Lambda_{0}$ ) plays the role of complex numbers. Throughout the paper, we employ the language of linear algebra consistently with these choices for the basic sets of scalars. In particular, the term "space" generally means a module over $\Lambda_{0}$ or a $\mathbb{Z}_{2}$-graded module over $\Lambda$; a "linear map" between two spaces means a $\Lambda_{0}$ or $\Lambda$-linear map. In dealing with $\mathbb{Z}_{2}$-graded structures, we always denote with $\operatorname{deg}(U)$ the degree, i.e., the parity of an object $U ; \operatorname{deg}(U) \in \mathbb{Z}_{2}$.

1.2. Superanalysis. Setting up superanalysis requires that the superalgebra $L$ be topological (in an appropriate sense). We are mainly interested in calculus for functions of one even variable $x$ and one odd variable $\theta: x$ ranges over $L_{0}$ (or the torus $\left.L_{0} / \mathbb{Z}\right)$ and $\theta$ over $L_{1}$. The set of pairs $(x, \theta)$ is called the (1|1)-dimensional superspace. A superfield is a differentiable function of $x$ and $\theta$, with values in the complex superalgebra $\Lambda$; we say that a superfield is even (resp. odd) if it takes values in $\Lambda_{0}$ (resp. $\Lambda_{1}$ ). Apart from superfields, that are scalar valued functions, we also consider vector or matrix valued functions of $x$ and $\theta$. We employ the odd superderivative $D:=\theta \partial / \partial x+\partial / \partial \theta$, satisfying the identity $D^{2}=\partial / \partial x$, and we denote by $\int d x d \theta$ the integration over superspace. All functions are assumed to satisfy appropriate boundary conditions in the $x$ variable, so that one can integrate by parts without introducing boundary terms. 
The action of $D$ is denoted with a prime, and the action of $\partial / \partial x$ with a subscript $x$; so, for example, the third superderivative of a superfield $f=f(x, \theta)$ is $f^{\prime \prime \prime}=f_{x}^{\prime}$. For the sake of clarity, we recall a standard notational convention for superdifferential operators: if $f$ is a superfield, and $k$ an integer, the operator $D^{k} f$ is the composition of $D^{k}$ with the multiplication operator by $f$; this should not be confused with the $k^{\text {th }}$ superderivative of $f$, which is a superfield. The context allows to distinguish the operator from the superfield; in Tables 1-4, notations like $D f, D^{-1} f$, (or $\partial_{x} f, \partial_{x}^{-1} f$, etc.) always stand for operators. A general $\Lambda_{0}$-linear operator $A$ mapping superfields into superfields is said to be even (resp. odd) if it preserves (resp. changes) the parity of superfields; so, $\partial_{x}$ is even, $D$ is odd, and the multiplication operator by a superfield $f$ has the same parity as $f$. The adjoint of $A$ is the unique operator, denoted with $A^{*}$, such that

$$
\int d x d \theta f(A g)=(-1)^{\operatorname{deg}(f) \operatorname{deg}(A)} \int d x d \theta\left(A^{*} f\right) g
$$

for each pair of superfields $f$ and $g$. For the product of operators, it is found that $(A B)^{*}=(-1)^{\operatorname{deg}(A) \operatorname{deg}(B)} B^{*} A^{*}$, implying that $\left(A^{-1}\right)^{*}=(-1)^{\operatorname{deg}(A)}\left(A^{*}\right)^{-1}$ if $A$ is invertible. Moreover, $D^{*}=-D, \partial_{x}^{*}=-\partial_{x}$; if $f$ is a superfield of any parity, for the corresponding multiplication operator we have $f^{*}=f$.

1.3. Supermanifolds and tensors. The introductory remarks about supermanifolds and tensor fields given in [MP1] are also useful for our present purposes. If $\mathscr{M}$ is a supermanifold, and $m \in \mathscr{M}$, we denote by $T_{m} \mathscr{M}$ the (even) tangent space, and by $T_{m}^{*} \mathscr{M}$ the (even) cotangent space (both of them are $\Lambda_{0}$-modules). Tangent vectors and covectors will often be indicated, respectively, by $\dot{m}$ and $\delta m$, and the pairing by $\langle\delta m, \dot{m}\rangle$. A vector, or covector field is a section of the (even) tangent, or cotangent bundle.

The phase spaces of the susy AKNS theory and the other systems considered in this paper are infinite-dimensional supermanifolds of maps, where each point is a function on (1|1)-dimensional superspace. Several types of tensors are considered on these manifolds. As is usual, the term $(2,0)$ tensor means a map $m \mapsto P_{m}$ where, for each $m \in \mathscr{M}, P_{m}$ is a linear operator from $T_{m}^{*} \mathscr{M}$ into $T_{m} \mathscr{M}$. A $(1,1)$ tensor is a map $m \mapsto N_{m}$, where $N_{m}$ is a linear operator of $T_{m} \mathscr{M}$ into itself. A $(0,2)$ tensor is a map $m \mapsto \Omega_{m}$, where $\Omega_{m}$ is a linear operator from $T_{m} \mathscr{M}$ to $T_{m}^{*} \mathscr{M}$. In the sequel, we will extensively work with Poisson tensors, recursion operators and (pre)symplectic tensors on $\mathscr{M}$. As usually, a Poisson tensor means a skew-symmetric $(2,0)$ tensor $P$ such that the bracket $\{f, l\}:=\langle d f, P d l\rangle(f, l$ even functions on $\mathscr{M})$ satisfies the Jacobi identity. A recursion operator is a $(1,1)$ tensor $N$ with vanishing Nijenhuis torsion and a (pre)symplectic tensor is a skew-symmetric $(0,2)$ tensor $\Omega$ with vanishing exterior derivative [FF, MMR, LiM].

1.4. Lie superalgebra gl(2,2) and associated loop superalgebra. The Lie superalgebra $g l(2,2, \Lambda)$ (denoted for brevity with $g l(2,2)$ ) consists of $4 \times 4$ matrices with entries in the set of scalars 1 . Any such matrix can be written in block form as $U=\left(\begin{array}{cc}X & Y \\ Z & W\end{array}\right)$, where each block is a $2 \times 2$ matrix. The even part of $g l(2,2)$, denoted by $g l(2,2)_{0}$, consists of matrices with even entries in the blocks $X, W$ and odd entries in $Y, Z$; similarly, the odd part $g l(2,2)_{1}$ consists of matrices with the parities of the blocks interchanged. $g l(2,2)$ can be regarded as a $\mathbb{Z}_{2}$-graded $\Lambda$-module, and it is a Lie superalgebra with the supercommutator $[U, V]:=U V-(-1)^{\operatorname{deg}(U) \operatorname{deg}(V)} V U$. 
The loop superalgebra $\mathscr{G}=g l(2,2)\{x, \theta\}$ consists of $g l(2,2)$ valued functions of the superspace variables $x$ and $\theta$. An element of $\mathscr{G}$ can be represented as a $4 \times 4$ matrix where each entry is a superfield depending on $x$ and $\theta$. $\mathscr{G}$ is a Lie superalgebra; the even and odd parts $\mathscr{G}_{0}, \mathscr{G}_{1}$, as well as the supercommutator [, ], are defined pointwisely in terms of the omologous structures of $g l(2,2)$. If $U=U(x, \theta)$ is an element of $\mathscr{G}$, written in terms of $2 \times 2$ blocks, we define $D U:=\left(\begin{array}{cc}D X & D Y \\ -D Z & -D W\end{array}\right)$. For further details about the topic of this subsection, see [Lei, Cor].

\section{The susy AKNS: Main Results}

2.1. The susy AKNS from the biHamiltonian viewpoint. Let us consider a (flat) supermanifold $\mathscr{M}$, whose points are quadruples $m=(b, a, \psi, \varphi)$, where $b=b(x, \theta)$ and $a=a(x, \theta)$ are even superfields, $\psi=\psi(x, \theta)$ and $\varphi=\varphi(x, \theta)$ are odd superfields. A tangent vector and a covector at any point $m$ are written, respectively, as $\dot{m}=(\dot{b}, \dot{a}, \dot{\psi}, \dot{\varphi})$ and $\delta m=(\delta b, \delta a, \delta \psi, \delta \varphi)$, where $\dot{b}, \dot{a}, \delta \psi, \delta \varphi$ are even superfields and $\dot{\psi}, \dot{\varphi}, \delta b, \delta a$ are odd; we have the pairing $\langle\delta m, \dot{m}\rangle:=\int d x d \theta(\delta b \dot{b}+\delta a \dot{a}+$ $\delta \psi \dot{\psi}+\delta \varphi \dot{\varphi})$.

The manifold $\mathscr{M}$ carries a pair of Poisson tensors $Q, P$, which are compatible in the sense of [Ma1]; so, the triple $(\mathscr{M}, Q, P)$ is a biHamiltonian supermanifold. The explicit expressions for $Q$ and $P$ are given in Table 1a, which also contains the expression of the symplectic tensor $\Omega:=Q^{-1}$.

We remark that $Q$ and $P$ are skew symmetric in the ordinary sense, i.e., $\left\langle\delta m, Q_{m} \delta m^{\prime}\right\rangle=-\left\langle\delta m^{\prime}, Q_{m} \delta m\right\rangle$ and similarly for $P$. If we introduce the matrix elements $Q_{i k}$, as in Table 1a (with $i$ and $k$ ranging through the symbols $b, a, \psi, \varphi$ ), from the skew-symmetry of $Q$ we infer

$$
Q_{k i}=-(-1)^{\operatorname{deg}(i) \operatorname{deg}(k)+\operatorname{deg}(i)+\operatorname{deg}(k)}\left(Q_{i k}\right)^{*},
$$

where the r.h.s. contains the adjoint of $Q_{i k}$ defined according to Subsect. 1.2; the same holds for the matrix elements of $P$.

Similarly, the skew-symmetry property $\left\langle\dot{m}, \Omega_{m} \dot{m}^{\prime}\right\rangle=-\left\langle\dot{m}^{\prime}, \Omega_{m} \dot{m}\right\rangle$ implies

$$
\Omega_{k i}=(-1)^{\operatorname{deg}(i) \operatorname{deg}(k)+1}\left(\Omega_{i k}\right)^{*} .
$$

We now describe the susy AKNS hierarchy. Let us consider the vector field $K_{0}(m)=m_{x}$, i.e., the generator of translations in the even variable $x$. It turns out that $K_{0}$ is Hamiltonian with respect to both Poisson tensors $Q$ and $P$; indeed, if we define the Hamiltonian functions $h_{0}(m):=-\int d x d \theta\left(b \varphi+a \psi+\psi \varphi^{\prime}\right)$, $h_{1}(m):=\frac{1}{2} \int d x d \theta\left(b \varphi_{x}+\psi^{\prime} \varphi_{x}-a \psi_{x}\right)$, we find that $K_{0}=P d h_{0}=Q d h_{1}(d$ denoting the differential). The general theory of biHamiltonian manifolds implies that there exists a hierarchy of vector fields $K_{j}$ and Hamiltonian functions $h_{j}$ satisfying the recursion relations

$$
K_{j}=P d h_{j}=Q d h_{j+1}, \quad(j=0,1,2, \ldots) .
$$

This is, by definition, the susy AKNS hierarchy(see the summary in Table 1a, where we also include the vector field $K_{-1}:=Q d h_{0}$ ). In spite of the nonlocal character of the Poisson tensors, the vector fields and the Hamiltonians appear to be local. 
As is known from the theory of biHamiltonian systems, the vector fields $K_{j}$ mutually commute and each Hamiltonian function is a constant of motion for all the vector fields of the hierarchy; also, the Hamiltonians are in involution with respect to both Poisson brackets induced by $Q$ and $P:\left\langle d h_{j}, Q d h_{j^{\prime}}\right\rangle=\left\langle d h_{j}, P d h_{j^{\prime}}\right\rangle=0$ for each $j, j^{\prime}$.

Equation (2.1.3) implies

$$
K_{j+1}=N K_{j} \quad(j=0,1,2, \ldots),
$$

where $N:=P \circ Q^{-1}$. This is a recursion operator, i.e., a $(1,1)$ type tensor with vanishing Nijenhuis torsion. The $(0,2)$ type tensor $\Omega=Q^{-1}$ is symplectic; $\Omega$ and $N$ are compatible, i.e., the $(0,2)$ tensor $\Omega \circ N$ is itself (pre)symplectic.

Equation (2.1.3) also implies

$$
d h_{j+1}=N^{*} d h_{j} \quad(j=0,1,2, \ldots),
$$

where $N^{*}$ is the adjoint of $N$. At each point $m, N_{m}^{*}$ sends $T_{m}^{*} \mathscr{M}$ into itself and is defined by the condition $\left\langle\delta m, N_{m} \dot{m}\right\rangle=\left\langle N_{m}^{*} \delta m, \dot{m}\right\rangle$.

The explicit expression of $N$ is given in Table 1a; here we report all matrix elements $N_{i k}$ for $i, k$ ranging within the symbols $b, a, \psi, \varphi$. The adjoint $N^{*}$ can be described similarly, specifying a list of matrix elements $\left(N^{*}\right)_{k i}$; on account of the previous definition, we find

$$
\left(N^{*}\right)_{k i}=(-1)^{\operatorname{deg}(k)(\operatorname{deg}(i)+1)}\left(N_{i k}^{*}\right),
$$

where the r.h.s contains the adjoint of $N_{i k}$ defined according to Subsect. 1.2.

As will be shown in Subsect. 3.1, the classical AKNS theory can be obtained by restriction of the susy theory to the "classical" submanifold $\mathscr{M}_{\mathrm{cl}}:=\{(b, a, \psi, \varphi) \in \mathscr{M} \mid$ $b=0, a=0, \psi(x, \theta)=\theta q(x), \varphi(x, \theta)=\theta r(x)\} \quad(q, r$ arbitrary even functions of $x)$.

Remark. Equations (2.1.4-5) provide an efficient scheme for practical computation of the hierarchy. Let us illustrate this fact by considering, for example, the recursion relation for the Hamiltonians. In order to apply it, we need the explicit expression for $N^{*}$, which can be obtained from Table la and from the adjunction rules given above. If $\delta m=(\delta b, \delta a, \delta \psi, \delta \varphi)$ is any covector at $m$, it is found that the covector $\widehat{\delta m}:=N_{m}^{*} \delta m$ has components

$$
\begin{aligned}
& \widehat{\delta b}=\frac{1}{2}\left(-\delta b_{x}+\psi \varphi \delta b+a \varepsilon+\varphi^{\prime} \varepsilon+a \eta+\varphi s\right), \\
& \widehat{\delta a}=\frac{1}{2}\left(\delta a_{x}+\psi \varphi \delta a++b \varepsilon+\psi^{\prime} \eta+b \eta+\psi s\right), \\
& \widehat{\delta \psi}=\frac{1}{2}\left(-\delta \psi_{x}+\psi \varphi \delta \varphi+a^{\prime} \eta+a g+\varphi^{\prime} g+\varphi \rho\right), \\
& \widehat{\delta \varphi}=\frac{1}{2}\left(\delta \varphi_{x}+\psi \varphi \delta \varphi+b^{\prime} \varepsilon+b g+\psi^{\prime} g+\psi \sigma\right),
\end{aligned}
$$

where the odd superfields $\varepsilon, \eta, \rho, \sigma$ and the even superfields $s, g$ are such that:

$$
\begin{aligned}
& \varepsilon_{x}=\left(b+\psi^{\prime}\right) \delta b-\psi \delta b^{\prime}-a \delta a+\psi \delta \psi-\varphi \delta \varphi \\
& \eta_{x}=b \delta b-\left(a+\varphi^{\prime}\right) \delta a+\varphi \delta a^{\prime}+\psi \delta \psi-\varphi \delta \varphi \\
& \rho_{x}=-b_{x} \delta b-a^{\prime} \delta a^{\prime}-\left(\psi_{x}+b^{\prime}\right) \delta \psi-b \delta \psi^{\prime}+a^{\prime} \delta \varphi+\left(a+\varphi^{\prime}\right) \delta \varphi^{\prime} \\
& \sigma_{x}:=b^{\prime} \delta b^{\prime}+a_{x} \delta a-b^{\prime} \delta \psi-\left(b+\psi^{\prime}\right) \delta \psi^{\prime}+\left(\varphi_{x}+a^{\prime}\right) \delta \varphi+a \delta \varphi^{\prime} \\
& s_{x}=-b \delta b^{\prime}+a \delta a^{\prime}+\left(2 b+\psi^{\prime}\right) \delta \psi-\left(2 a+\varphi^{\prime}\right) \delta \varphi \\
& g_{x}=b^{\prime} \delta b-a^{\prime} \delta a+2\left(b+\psi^{\prime}\right) \delta \psi-\psi \delta \psi^{\prime}-2\left(a+\varphi^{\prime}\right) \delta \varphi+\varphi \delta \varphi^{\prime}
\end{aligned}
$$


We are interested in the application of these formulas for constructing the Hamiltonian functions of the hierarchy. To this purpose, let us consider the covector field $d_{0}=d h_{0}$, which is the differential of the first Hamiltonian; at any point $m$ we have $d_{0}(m)=\left(-\varphi,-\psi,-a-\varphi^{\prime},-b-\psi^{\prime}\right)$. The covector field $d_{1}:=N^{*} d_{0}$ is the differential of the Hamiltonian $h_{1}$. Consider the sequence of covector fields defined by $d_{j+1}=N^{*} d_{j}$; by a general property of recursion operators, the covector fields constructed in this way are exact, i.e., there exist functions $h_{j}$ such that $d_{j}=d h_{j}$.

For constructing the covectors $d_{j}(m)$ at a point, one computes, at each step of the iteration, the functions $\varepsilon, \eta, \ldots, g$ whose $x$-derivatives are given by Eqs. (2.1.8); for $j=0,1,2, \ldots$ it is found that the right-hand sides of these equations are total derivatives, so the covector fields and the Hamiltonians determined in this way are local functions of $m$. An analysis of this kind can be carried out also for the recursion relation (2.1.4) of the vector fields.

2.2. The zero curvature/Lax formulation for the susy AKNS. Let us consider the loop superalgebra $\mathscr{G}=g l(2,2)\{x, \theta\}$, already introduced in Subsect. 1.4; the elements of $\mathscr{G}$ are $4 \times 4$ matrices, whose entries are superfields. We denote by $\mathscr{G}^{\lambda}$, $\mathscr{G}_{0}^{\lambda}$ and $\mathscr{G}_{1}^{\lambda}$ the sets of polynomials in one (even) parameter $\lambda$ and coefficients in $\mathscr{G}$, in the even part $\mathscr{G}_{0}$ and in the odd part $\mathscr{G}_{1}$, respectively; each of these polynomials can be represented as a matrix where the entries depend on $\lambda$. We exhibit a family of maps $\mathscr{M} \rightarrow \mathscr{G}_{1}^{\lambda}, m \mapsto \Sigma^{\lambda}(m)$ and $\mathscr{M} \rightarrow \mathscr{G}_{0}^{\lambda}, m \mapsto \mathscr{C}_{j}^{\lambda}(m) \quad(j=-1,0,1, \ldots)$ such that, for each vector field $K_{j}$ of the susy AKNS, the following holds:

$$
\frac{d \Sigma^{\lambda}(m)}{d t_{j}}-D \mathscr{C}_{j}^{\lambda}(m)=\left[\Sigma^{\lambda}(m), \mathscr{C}_{j}^{\lambda}(m)\right]
$$

$\left(d / d t_{j}\right.$ denoting the derivative along $\left.K_{j}\right)$. We call this equation the zero curvature representation of the susy AKNS hierarchy. Explicitly, we have

$$
\Sigma^{\lambda}(b, a, \psi, \varphi):=\left(\begin{array}{cccc}
0 & -\frac{1}{2} \psi & -1 & 0 \\
-\frac{1}{2} \varphi & 0 & 0 & -1 \\
\frac{1}{4} \varphi \psi-\lambda & -b-\frac{1}{2} \psi^{\prime} & 0 & -\frac{1}{2} \psi \\
-a-\frac{1}{2} \varphi^{\prime} & -\frac{1}{4} \varphi \psi+\lambda & -\frac{1}{2} \varphi & 0
\end{array}\right)
$$

(note that the time derivative of $\Sigma^{\lambda}(m)$ is independent of $\lambda$ ). The matrices $\mathscr{C}_{j}^{\lambda}(m)$ for the lowest values of $j$ are reported in Table 1b. In principle, each of these matrices can be computed requiring that:

i) it is a polynomial of degree $j+1$ in $\lambda$ with leading term $(-1)^{j+1} \lambda^{j+1} \mathscr{Z}$, where $\mathscr{Z}$ is the constant diagonal matrix $\operatorname{Diag}(-1,1,-1,1)$;

ii) the sum $D \mathscr{C}_{j}^{\lambda}(m)+\left[\Sigma^{\lambda}(m), \mathscr{C}_{j}^{\lambda}(m)\right]$ is independent of $\lambda$.

One could interpret Eq. (2.2.1) as an alternative definition of the susy AKNS hierarchy. According to this viewpoint, after constructing the matrices $\mathscr{C}_{j}$ with the requirements i) and ii), one defines $K_{j}$ as the unique vector field such that Eq. (2.2.1) holds. On the contrary, in our approach the hierarchy is defined by the biHamiltonian recursion scheme of Subsect. 2.1 and the zero curvature formulation is a supplementary output. 
The biHamiltonian approach allows a geometrical interpretation for the matrices $\mathscr{C}_{j}[\mathrm{MP} 2]$ and provides the following formula for their construction:

$$
\mathscr{C}_{j}^{\lambda}(m)=\sum_{k=0}^{j}(-1)^{k} \lambda^{k} \Sigma^{\star \lambda}\left(m, d_{j-k}(m)\right)+(-1)^{j+1} \lambda^{j+1} \mathscr{Z} .
$$

Here, $\Sigma^{\star \lambda}$ is a map, reported in Table $1 \mathrm{~b}$, which associates to each covector on the phase space $\mathscr{M}$ an element of $\mathscr{G}_{0}^{\lambda}$; the covector fields $d_{j}$, which are computed recursively using the tensor $N^{*}$, are the differentials of the Hamiltonian functions $h_{j}$.

As is usual in the classical framework [DS], we can interpret the zero curvature representation as a Lax formulation, where the Lax operator is a matrix first order differential operator. To this purpose, let us introduce a space $\mathscr{W}$ of quadruples $\Phi=$ $\left(\begin{array}{c}\Psi_{1} \\ \Psi_{2} \\ \xi_{1} \\ \xi_{2}\end{array}\right)$, where the components $\Psi_{i}=\Psi_{i}(x, \theta)$ and $\xi_{i}=\xi_{i}(x, \theta)$ are superfields. An element of $\mathscr{W}$ is defined to be even if the superfields $\Psi_{i}$ are even and the $\xi_{i}$ are odd; the odd elements of $\mathscr{W}$ are defined similarly, interchanging the parities of $\Psi_{i}, \xi_{i}$. The superderivative $D$ acts on $\mathscr{W}$ according to the rule $D \Phi:=\left(\begin{array}{c}D \Psi_{1} \\ D \Psi_{2} \\ -D \xi_{1} \\ -D \xi_{2}\end{array}\right)$; we also observe that the elements of the loop algebra $\mathscr{G}$ act naturally on $\mathscr{W}$ by matrix multiplication.

Let $\operatorname{Lin}(\mathscr{W})$ denote the set of linear operators on $\mathscr{W}$, and $\operatorname{Lin}^{2}(\mathscr{W})$ the set of polynomials in one variable $\lambda$, whose coefficients belong to $\operatorname{Lin}(\mathscr{W})$; each element $\mathscr{A}^{\lambda}$ of $\operatorname{Lin}^{\lambda}(\mathscr{W})$ is in fact an operator on $\mathscr{W}$, depending polynomially on the parameter $\lambda: \mathscr{A}^{\lambda}=\mathscr{A}_{0}+\lambda A_{1}+\cdots+\lambda^{n} \mathscr{A}_{n}$, where $\mathscr{A}_{0}, \ldots, \mathscr{A}_{n}$ are operators. We define a map $\mathscr{M} \rightarrow \operatorname{Lin}^{\lambda}(\mathscr{W}), m \mapsto \mathscr{L}^{\lambda}(m)$ setting

$$
\begin{aligned}
\mathscr{L}^{\lambda}(b, a, \psi, \varphi) & :=D+\Sigma^{\lambda}(m)= \\
& =\left(\begin{array}{cccc}
D & -\frac{1}{2} \psi & -1 & 0 \\
-\frac{1}{2} \varphi & D & 0 & -1 \\
\frac{1}{4} \varphi \psi-\lambda & -b-\frac{1}{2} \psi^{\prime} & -D & -\frac{1}{2} \psi \\
-a-\frac{1}{2} \varphi^{\prime} & -\frac{1}{4} \varphi \psi+\lambda & -\frac{1}{2} \varphi & -D
\end{array}\right) .
\end{aligned}
$$

It is straightforward to check that the zero curvature representation (2.2.1) is equivalent to the Lax formulation

$$
\frac{d \mathscr{L}^{\lambda}(m)}{d t_{j}}=\left[\mathscr{L}^{\lambda}(m), \mathscr{C}_{j}^{\lambda}(m)\right],
$$

containing the ordinary commutator between $\mathscr{L}^{\lambda}(m)$ and $\mathscr{C}_{j}^{\lambda}(m)$ as $\lambda$-dependent operators on $\mathscr{W}$.

The spectral problem associated to the Lax operator $\mathscr{L}^{\lambda}(m)$ is the equation $\mathscr{L}^{\lambda}(m) \Phi^{\lambda}=0$, where $\Phi^{\lambda}=\left(\begin{array}{c}\Psi_{1}^{\lambda} \\ \psi_{2}^{\lambda} \\ \xi_{1}^{\lambda} \\ \xi_{2}^{\lambda}\end{array}\right)$ is a vector of $\mathscr{W}$, depending on the parameter $\lambda$. Using Eq. (2.2.4), we find that the spectral problem is equivalent to

$$
\begin{array}{cc}
-\Psi_{1, x}^{\lambda}-(b+\psi D) \Psi_{2}^{\lambda}=\lambda \Psi_{1}^{\lambda}, & \Psi_{2, x}^{\lambda}+(a+\varphi D) \Psi_{1}^{\lambda}=\lambda \Psi_{2}^{\lambda}, \\
\xi_{1}^{\lambda}=D \Psi_{1}^{\lambda}-\frac{1}{2} \psi \Psi_{2}, & \xi_{2}^{\lambda}=D \Psi_{2}^{\lambda}-\frac{1}{2} \varphi \Psi_{1} .
\end{array}
$$


The first two equations define an eigenvalue problem

$$
L(m)\left(\begin{array}{l}
\Psi_{1}^{\lambda} \\
\Psi_{2}^{\lambda}
\end{array}\right)=\lambda\left(\begin{array}{l}
\Psi_{1}^{\lambda} \\
\Psi_{2}^{\lambda}
\end{array}\right), \quad L(m):=\left(\begin{array}{cc}
-\partial_{x} & -b-\psi D \\
a+\varphi D & \partial_{x}
\end{array}\right) .
$$

The operator $L(m)$ acts on a space $\mathscr{H}$ of pairs $\left(\begin{array}{l}\Psi_{1}(x, \theta) \\ \Psi_{2}(x, \theta)\end{array}\right)$; it can employed to set up a reduced, parameter independent Lax formulation of the susy AKNS in terms of $2 \times 2$ matrix differential operators. In fact, we have

$$
\frac{d L}{d t_{j}}(m)=\left[L(m), C_{j}(m)\right]
$$

where the operators $C_{j}(m)$ are constructed as explained in Table $1 \mathrm{~b}$.

Finally, we recall a general property of the Lax formalism, which will be useful in Sect. 3: each power of the operators $\mathscr{L}^{\lambda}(m)$ and $L(m)$ can be as well employed to set up a Lax formulation. In particular, if we consider the squares we get

$$
\begin{aligned}
\frac{d\left(\mathscr{L}^{\lambda}\right)^{2}(m)}{d t_{j}} & =\left[\left(\mathscr{L}^{\lambda}\right)^{2}(m), \mathscr{C}_{j}^{\lambda}(m)\right], \\
\frac{d L^{2}(m)}{d t_{j}} & =\left[L^{2}(m), C_{j}(m)\right] .
\end{aligned}
$$

Table 1a. The susy AKNS: biHamiltonian formulation

First Poisson tensor $Q_{m}: T_{m}^{*} \mathscr{M} \rightarrow T_{m} \mathscr{M}$ and its inverse $\Omega_{m}:=Q_{m}^{-1}$ at a point $m=(b, a, \psi, \varphi)$ :

$$
\begin{aligned}
Q_{m}\left(\begin{array}{c}
\delta b \\
\delta a \\
\delta \psi \\
\delta \varphi
\end{array}\right) & =2\left(\begin{array}{cccc}
Q_{b b} & Q_{b a} & Q_{b \psi} & Q_{b \varphi} \\
Q_{a b} & Q_{a a} & Q_{a \psi} & Q_{a \varphi} \\
Q_{\psi b} & Q_{\psi a} & 0 & 0 \\
Q_{\varphi b} & Q_{\varphi a} & 0 & 0
\end{array}\right)\left(\begin{array}{c}
\delta b \\
\delta a \\
\delta \psi \\
\delta \varphi
\end{array}\right), \\
\Omega_{m}\left(\begin{array}{c}
\dot{b} \\
\dot{a} \\
\dot{\psi} \\
] \dot{\varphi}
\end{array}\right) & =\frac{1}{2}\left(\begin{array}{cccc}
0 & 0 & \Omega_{b \psi} & \Omega_{b \varphi} \\
0 & 0 & \Omega_{a \psi} & \Omega_{a \varphi} \\
\Omega_{\psi b} & \Omega_{\psi a} & \Omega_{\psi \psi} & \Omega_{\psi \varphi} \\
\Omega_{\varphi b} & \Omega_{\varphi a} & \Omega_{\varphi \psi} & \Omega_{\varphi \varphi}
\end{array}\right)\left(\begin{array}{c}
\dot{b} \\
\dot{a} \\
\dot{\psi} \\
\dot{\varphi}
\end{array}\right),
\end{aligned}
$$

$$
\begin{array}{ll}
Q_{b b}:=\psi \partial_{x}^{-1} b-b \partial_{x}^{-1} \psi, & \Omega_{b \psi}:=\varphi \partial_{x}^{-1} \varphi, \\
Q_{b a}:=D-\psi \partial_{x}^{-1}(a+D \varphi)-b \partial_{x}^{-1} \varphi, & \Omega_{b \varphi}:=1-\varphi \partial_{x}^{-1} \psi, \\
Q_{b \psi}:=\psi \partial_{x}^{-1} \psi, & \Omega_{a \psi}:=-1+\psi \partial_{x}^{-1} \varphi, \\
Q_{b \varphi}:=-1-\psi \partial_{x}^{-1} \varphi, & \Omega_{a \varphi}:=-\psi \partial_{x}^{-1} \psi, \\
Q_{a a}:=-\varphi \partial_{x}^{-1} a+a \partial_{x}^{-1} \varphi, & \Omega_{\psi \psi}:=-\varphi \partial_{x}^{-1}\left(a+\varphi^{\prime}\right)+\left(a+\varphi^{\prime}\right) \partial_{x}^{-1} \varphi, \\
Q_{a \psi}:=1+\varphi \partial_{x}^{-1} \psi, & \Omega_{\psi \varphi}:=D-\varphi \partial_{x}^{-1}(b+\psi D)-\left(a+\varphi^{\prime}\right) \partial_{x}^{-1} \psi, \\
Q_{a \varphi}:=-\varphi \partial_{x}^{-1} \varphi, & \Omega_{\varphi \varphi}:=\psi \partial_{x}^{-1}\left(b+\psi^{\prime}\right)-\left(b+\psi^{\prime}\right) \partial_{x}^{-1} \psi, \\
Q_{a b}=\left(Q_{b a}\right)^{*}, Q_{\psi b}=-\left(Q_{b \psi}\right)^{*}, \text { etc. } & \Omega_{\psi b}=-\left(\Omega_{b \psi}\right)^{*}, \Omega_{\varphi \psi}=\left(\Omega_{\psi \varphi}\right)^{*}, \text { etc } .
\end{array}
$$


Second Poisson tensor at a point $m$ :

$$
P_{m}: T_{m}^{*} \mathscr{M} \rightarrow T_{m} \mathscr{M}, \quad P_{m}\left(\begin{array}{c}
\delta b \\
\delta a \\
\delta \psi \\
\delta \varphi
\end{array}\right)=\left(\begin{array}{cccc}
P_{b b} & P_{b a} & P_{b \psi} & P_{b \varphi} \\
P_{a b} & P_{a a} & P_{a \psi} & P_{a \varphi} \\
P_{\psi b} & P_{\psi a} & P_{\psi \psi} & P_{\psi \varphi} \\
P_{\varphi b} & P_{\varphi a} & P_{\varphi \psi} & P_{\varphi \varphi}
\end{array}\right)\left(\begin{array}{c}
\delta b \\
\delta a \\
\delta \psi \\
\delta \varphi
\end{array}\right),
$$

$$
\begin{aligned}
P_{b b}:= & 2 b \psi-b \partial_{x}^{-1}\left(2 b \varphi+2 a \psi+\psi \varphi^{\prime}+\varphi \psi^{\prime}\right) \partial_{x}^{-1} b-b \partial_{x}^{-1}\left(b \varphi+a \psi+\psi \varphi^{\prime}\right) D^{-1} \psi \\
& +b \partial_{x}^{-1}\left(b^{\prime}-\psi x+2 b D\right)-\psi D^{-1}\left(b \varphi+a \psi+\psi \varphi^{\prime}\right) \partial_{x}^{-1} b+\left(b^{\prime}+\psi\right) \partial_{x}^{-1} b \\
P_{b a}:= & D \partial_{x}-2 \varphi \psi D-2\left(b \varphi+a \psi+\psi \varphi^{\prime}\right)+b \partial_{x}^{-1}\left(2 b \varphi+2 a \psi+\psi \varphi^{\prime}+\varphi \psi^{\prime}\right) \partial_{x}^{-1} a \\
& +b \partial_{x}^{-1}\left(b \varphi+a \psi+\psi^{\prime} \varphi\right) D^{-1} \varphi+b \partial_{x}^{-1}\left(\varphi_{x}-a^{\prime}-2 a D\right)+\psi D^{-1}\left(a^{\prime}+\varphi_{x}\right) \\
& +\psi D^{-1}\left(b \varphi+a \psi+\psi \varphi^{\prime}\right) \partial_{x}^{-1}(a+D \varphi)-\left(b^{\prime}+\psi x \partial_{x}^{-1}(a+D \varphi)\right. \\
P_{b \psi}:= & -b \partial_{x}^{-1}\left(2 b \varphi+2 a \psi+\psi \varphi^{\prime}+\varphi \psi^{\prime}\right) \partial_{x}^{-1} \psi-b \partial_{x}^{-1}(2 b+\psi D)+\left(b^{\prime}+\psi_{x}\right) \partial_{x}^{-1} \psi \\
& -\psi D^{-1} b-\psi D^{-1}\left(b \varphi+a \psi+\psi \varphi^{\prime}\right) \partial_{x}^{-1} \psi, \\
P_{b \varphi}:= & -\partial_{x}+2 \varphi \psi+b \partial_{x}^{-1}(2 a+\varphi D)+b \partial_{x}^{-1}\left(2 b \varphi+2 a \psi+\psi \varphi^{\prime}+\varphi \psi^{\prime}\right) \partial_{x}^{-1} \varphi \\
& +\psi D^{-1}\left(b \varphi+a \psi+\psi \varphi^{\prime}\right) \partial_{x}^{-1} \varphi+\psi D^{-1}\left(a+\varphi^{\prime}\right)-\left(b^{\prime}+\psi x\right) \partial_{x}^{-1} \varphi \\
P_{a a}:= & 2 a \varphi-a \partial_{x}^{-1}\left(2 b \varphi+2 a \psi+\psi \varphi^{\prime}+\varphi \psi^{\prime}\right) \partial_{x}^{-1} a-a \partial_{x}^{-1}\left(b \varphi+a \psi+\varphi \psi^{\prime}\right) D^{-1} \varphi \\
& +a \partial_{x}^{-1}\left(a^{\prime}-\varphi_{x}+2 a D\right)-\varphi D^{-1}\left(b \varphi+a \psi+\varphi \psi^{\prime}\right) \partial_{x}^{-1} a+\left(a^{\prime}+\varphi_{x}\right) \partial_{x}^{-1} a \\
P_{a \psi}:= & -\partial_{x}^{-2} 2 \psi+a \partial_{x}^{-1}(2 b+\psi D)+a \partial_{x}^{-1}\left(2 b \varphi+2 a \psi+\psi \varphi^{\prime}+\varphi \psi^{\prime}\right) \partial_{x}^{-1} \psi \\
& +\varphi D^{-1}\left(b \varphi+a \psi+\varphi \psi^{\prime}\right) \partial_{x}^{-1} \psi+\varphi D^{-1}\left(b+\psi^{\prime}\right)-\left(a^{\prime}+\varphi_{x}\right) \partial_{x}^{-1} \psi, \\
P_{a \varphi}:= & -a \partial_{x}^{-1}\left(2 b \varphi+2 a \psi+\psi \varphi^{\prime}+\varphi \psi^{\prime}\right) \partial_{x}^{-1} \varphi-a \partial_{x}^{-1}(2 a+\varphi D)+\left(a^{\prime}+\varphi_{x}\right) \partial_{x}^{-1} \varphi \\
& -\varphi D^{-1} a-\varphi D^{-1}\left(b \varphi+a \psi+\varphi \psi^{\prime}\right) \partial_{x}^{-1} \varphi, \\
P_{\psi \psi}:= & -\psi \partial_{x}^{-1}\left(2 b \varphi+2 a \psi+\psi \varphi^{\prime}+\varphi \psi^{\prime}\right) \partial_{x}^{-1} \psi-\psi \partial_{x}^{-1}\left(2 b+\psi^{\prime}\right)-\left(2 b+\psi^{\prime}\right) \partial_{x}^{-1} \psi \\
P_{\psi \varphi}:= & \psi \partial_{x}^{-1}\left(2 b \varphi+2 a \psi+\psi \varphi^{\prime}+\varphi \psi^{\prime}\right) \partial_{x}^{-1} \varphi+\psi \partial_{x}^{-1}\left(2 a+\varphi \varphi^{\prime}\right)+\left(2 b+\psi^{\prime}\right) \partial_{x}^{-1} \varphi \\
P_{\varphi \varphi}:= & -\varphi \partial_{x}^{-1}\left(2 b \varphi+2 a \psi+\psi \varphi^{\prime}+\varphi \psi^{\prime}\right) \partial_{x}^{-1} \varphi-\varphi \partial_{x}^{-1}\left(2 a+\varphi^{\prime}\right)-\left(2 a+\varphi^{\prime}\right) \partial_{x}^{-1} \varphi \\
P_{a b}= & -\left(P_{b a}\right)^{*}, \quad P_{\psi b}=\left(P_{b \psi}\right)^{*}, \quad P_{\varphi \psi}=-\left(P_{\psi \varphi}\right)^{*}, \text { etc. }
\end{aligned}
$$

Recursion operator $N_{m}: T_{m} \mathscr{M} \rightarrow T_{m} \mathscr{M}, \quad N_{m}\left(\begin{array}{c}\dot{b} \\ \dot{a} \\ \dot{\psi} \\ \dot{\varphi}\end{array}\right)=\frac{1}{2}\left(\begin{array}{cccc}N_{b b} & N_{b a} & N_{b \psi} & N_{b \varphi} \\ N_{a b} & N_{a a} & N_{a \psi} & N_{a \varphi} \\ N_{\psi b} & N_{\psi a} & N_{\psi \psi} & N_{\psi \varphi} \\ N_{\varphi b} & N_{\varphi a} & N_{\varphi \psi} & N_{\varphi \varphi}\end{array}\right)\left(\begin{array}{c}\dot{b} \\ \dot{a} \\ \dot{\psi} \\ \dot{\varphi}\end{array}\right)$,

$N_{b b}:=\partial_{x}+\psi \varphi-b \partial_{x}^{-1}(2 a+\varphi D)+b^{\prime} \partial_{x}^{-1} \varphi-\psi D^{-1}\left(a+\varphi^{\prime}\right)$,

$N_{b a}:=b^{\prime} \partial_{x}^{-1} \psi-b \partial_{x}^{-1}(2 b+\psi D)-\psi D^{-1} b$,

$N_{b \psi}:=b^{\prime} \partial_{x}^{-1}\left(a+\varphi^{\prime}\right)+b \partial_{x}^{-1} a^{\prime}-b_{x} \partial_{x}^{-1} \varphi$,

$N_{b \varphi}:=b \partial_{x}^{-1} b^{\prime}+b^{\prime} \partial_{x}^{-1}(b+\psi D)+\psi D^{-1} b^{\prime}+b_{x} \partial_{x}^{-1} \psi$,

$N_{a b}:=a \partial_{x}^{-1}(2 a+\varphi D)-a^{\prime} \partial_{x}^{-1} \varphi+\varphi D^{-1} a$,

$N_{a a}:=-\partial_{x}+\psi \varphi+a \partial_{x}^{-1}(2 b+\psi D)-a^{\prime} \partial_{x}^{-1} \psi+\varphi D^{-1}\left(b+\psi^{\prime}\right)$,

$N_{a \psi}:=-\varphi D^{-1} a^{\prime}-a^{\prime} \partial_{x}^{-1}(a+\varphi D)-a \partial_{x}^{-1} a^{\prime}-a_{x} \partial_{x}^{-1} \varphi$,

$N_{a \varphi}:=a_{x} \partial_{x}^{-1} \psi-a^{\prime} \partial_{x}^{-1}\left(b+\psi^{\prime}\right)-a \partial_{x}^{-1} b^{\prime}$,

$N_{\psi b}:=-\psi \partial_{x}^{-1}\left(2 a+\varphi^{\prime}\right)-\left(2 b+\psi^{\prime}\right) \partial_{x}^{-1} \varphi, \quad N_{\psi a}:=-\psi \partial_{x}^{-1}\left(2 b+\psi^{\prime}\right)-\left(2 b+\psi^{\prime}\right) \partial_{x}^{-1} \psi$,

$N_{\psi \psi}:=\partial_{x}+\psi \varphi-b \partial_{x}^{-1}\left(2 a+\varphi^{\prime}+\varphi D\right)-\psi^{\prime} \partial_{x}^{-1}\left(a+\varphi^{\prime}\right)-\psi \partial_{x}^{-1}\left(\varphi_{x}+a D+\varphi^{\prime} D\right)-\psi_{x} \partial_{x}^{-1} \varphi$,

$N_{\psi \varphi}:=-b \partial_{x}^{-1}\left(2 b+\psi^{\prime}+\psi D\right)-\psi^{\prime} \partial_{x}^{-1}(b+\psi D)-\psi \partial_{x}^{-1}\left(\psi_{x}+b D+\psi^{\prime} D\right)+\psi_{x} \partial_{x}^{-1} \psi$,

$N_{\varphi b}:=\varphi \partial_{x}^{-1}\left(2 a+\varphi^{\prime}\right)+\left(2 a+\varphi^{\prime}\right) \partial_{x}^{-1} \varphi, \quad N_{\varphi a}:=\varphi \partial_{x}^{-1}\left(2 b+\psi^{\prime}\right)+\left(2 a+\varphi^{\prime}\right) \partial_{x}^{-1} \psi$,

$N_{\varphi \psi}:=a \partial_{x}^{-1}\left(2 a+\varphi^{\prime}+\varphi D\right)+\varphi^{\prime} \partial_{x}^{-1}(a+\varphi D)+\varphi \partial_{x}^{-1}\left(\varphi_{x}+a D+\varphi^{\prime} D\right)-\varphi_{x} \partial_{x}^{-1} \varphi$,

$N_{\varphi \varphi}:=-\partial_{x}+\psi \varphi+a \partial_{x}^{-1}\left(2 b+\psi^{\prime}+\psi D\right)+\varphi^{\prime} \partial_{x}^{-1}\left(b+\psi^{\prime}\right)+\varphi \partial_{x}^{-1}\left(\psi_{x}+b D+\psi^{\prime} D\right)+\varphi_{x} \partial_{x}^{-1} \psi$.

Recursion schemes: $K_{-1}:=Q d h_{0}, \quad K_{j}=P d h_{j}=Q d h_{j+1}(j=0,1,2 \ldots)$, or $d h_{j+1}=N^{*} d h_{j}(j=0,1,2 \ldots), \quad K_{j+1}=N K_{j}(j=-1,0,1, \ldots)$. 
First vector fields: $K_{-1}(m)=\left(\begin{array}{c}2 b \\ -2 a \\ 2 \psi \\ -2 \varphi\end{array}\right), \quad K_{0}(m)=\left(\begin{array}{c}b_{x} \\ a_{x} \\ \psi_{x} \\ \varphi_{x}\end{array}\right)$,

$K_{1}(m)=\frac{1}{2}\left(\begin{array}{c}b_{x x}-2 \varphi \psi b_{x}-2 \psi b^{\prime} \varphi^{\prime}-2 b \psi a^{\prime}-2 b \varphi b^{\prime}-2 a \psi b^{\prime}-2 a b^{2} \\ -a_{x x}-2 \varphi \psi a_{x}+2 \varphi a^{\prime} \psi^{\prime}+2 b \varphi a^{\prime}+2 a \psi a^{\prime}+2 a \varphi b^{\prime}+2 a^{2} b \\ \psi_{x x}-2 \varphi \psi \psi_{x}-2 \psi \varphi^{\prime} \psi^{\prime}-2 b \psi \varphi^{\prime}-2 b \varphi \psi^{\prime}-2 a \psi \psi^{\prime}-2 b^{2} \varphi-4 a b \psi \\ -\varphi_{x x}-2 \varphi \psi \varphi_{x}+2 \varphi \varphi^{\prime} \psi^{\prime}+2 b \varphi \varphi^{\prime}+2 a \psi \varphi^{\prime}+2 a \varphi \psi^{\prime}+4 a b \varphi+2 a^{2} \psi\end{array}\right)$,

$K_{2}(m)=\frac{1}{4}\left(\begin{array}{c}b_{x x x}-3 \varphi \psi b_{x x}-3 \varphi b_{x} \psi_{x}+3 b^{\prime} \varphi^{\prime} \psi_{x}-3 \psi a^{\prime} b_{x}-3 \varphi b^{\prime} b_{x}+3 b a^{\prime} \psi_{x} \\ +3 a b^{\prime} \psi_{x}-3 b \varphi b_{x}^{\prime}-3 a \psi b_{x}^{\prime}-3 \psi \varphi^{\prime} b_{x}^{\prime}-6 a b b_{x} \\ a_{x x x}+3 \varphi \psi a_{x x}-3 \psi a_{x} \varphi_{x}+3 a^{\prime} \psi^{\prime} \varphi_{x}-3 \psi a^{\prime} a_{x}-3 \varphi b^{\prime} a_{x}+3 b a^{\prime} \varphi_{x} \\ +3 a b^{\prime} \varphi_{x}-3 b \varphi a_{x}^{\prime}-3 a \psi a_{x}^{\prime}-3 \varphi \psi^{\prime} a_{x}^{\prime}-6 a b a_{x} \\ \psi_{x x x}-3 \varphi \psi \psi_{x x}-3 \varphi^{\prime} \psi^{\prime} \psi_{x}-3 \psi \varphi^{\prime} b_{x}-3 \varphi \psi^{\prime} b_{x}-6 a b \psi_{x}-3 b \varphi^{\prime} \psi_{x} \\ -3 a \psi^{\prime} \psi_{x}-3 b \varphi \psi_{x}^{\prime}-3 a \psi \psi_{x}^{\prime}-3 \psi \varphi^{\prime} \psi_{x}^{\prime}-6 b \varphi b_{x}-6 a \psi b_{x} \\ \varphi_{x x x}+3 \varphi \psi \varphi_{x x}-3 \varphi^{\prime} \psi^{\prime} \varphi_{x}-3 \psi \varphi^{\prime} a_{x}-3 \varphi \psi^{\prime} a_{x}-6 a b \varphi_{x}-3 b \varphi^{\prime} \varphi_{x} \\ -3 a \psi^{\prime} \varphi_{x}-3 b \varphi \varphi_{x}^{\prime}-3 a \psi \varphi_{x}^{\prime}-3 \varphi \psi^{\prime} \varphi_{x}^{\prime}-6 b \varphi a_{x}-6 a \psi a_{x}\end{array}\right)$.

First Hamiltonian functions: $h_{0}(m)=-\int d x d \theta\left(b \varphi+a \psi+\psi \varphi^{\prime}\right)$,

$h_{1}(m)=\frac{1}{2} \int d x d \theta\left(b \varphi_{x}+\psi^{\prime} \varphi_{x}-a \psi_{x}\right)$,

$h_{2}(m)=\frac{1}{4} \int d x d \theta\left(2 a b^{2} \varphi+2 a^{2} b \psi+b^{2} \varphi \varphi^{\prime}+2 a b \psi \varphi^{\prime}+2 a b \varphi \psi^{\prime}+a^{2} \psi \psi^{\prime}+2 b \varphi \varphi^{\prime} \psi^{\prime}\right.$

$\left.+2 a \psi \varphi^{\prime} \psi^{\prime}+\varphi \varphi^{\prime} \psi^{\prime 2}-b \varphi \psi \varphi_{x}-\varphi \psi \psi^{\prime} \varphi_{x}+a \varphi \psi \psi_{x}+\psi_{x} \varphi_{x}^{\prime}+b_{x} \varphi_{x}+a_{x} \psi_{x}\right)$.

Table 1b. The susy AKNS: Lax formalism

Parameter dependent Lax formulation: $d \mathscr{L}^{\lambda}(m) / d t_{j}=\left[\mathscr{L}^{\lambda}(m), \mathscr{C}_{j}^{\lambda}(m)\right], \quad(j=-1,0,1, \ldots)$ :

$\mathscr{L}^{\lambda}(m)=\left(\begin{array}{cccc}D & -\frac{1}{2} \psi & -1 & 0 \\ -\frac{1}{2} \varphi & D & 0 & -1 \\ \frac{1}{4} \varphi \psi-\lambda & -b-\frac{1}{2} \psi^{\prime} & -D & -\frac{1}{2} \psi \\ -a-\frac{1}{2} \varphi^{\prime} & -\frac{1}{4} \varphi \psi+\lambda & -\frac{1}{2} \varphi & -D\end{array}\right)$,

$\mathscr{C}_{j}^{\lambda}(m)=\sum_{k=0}^{J}(-1)^{k} \lambda^{k} \Sigma^{\star \lambda}\left(m, d_{j-k}(m)\right)+(-1)^{j+1} \lambda^{j+1} \mathscr{Z}$, where:

$\mathscr{Z}=\operatorname{Diag}(-1,1,-1,1)$,

$d_{0}(m)=\left(-\varphi,-\psi,-a-\varphi^{\prime},-b-\psi^{\prime}\right), \quad d_{j+1}(m)=N_{m}^{*} d_{j}(m), \quad(j=0,1,2, \ldots)$.

$\Sigma^{* \lambda}(m, \delta m)=\frac{1}{4}\left(\begin{array}{llll}\sigma_{11} & \sigma_{12} & \sigma_{13} & \sigma_{14} \\ \sigma_{21} & \sigma_{22} & \sigma_{23} & \sigma_{24} \\ \sigma_{31} & \sigma_{32} & \sigma_{33} & \sigma_{34} \\ \sigma_{41} & \sigma_{42} & \sigma_{43} & \sigma_{44}\end{array}\right)-\lambda\left(\begin{array}{cccc}w & 0 & 0 & 0 \\ 0 & w & 0 & 0 \\ w^{\prime}-\eta & 0 & w & 0 \\ 0 & 2 w^{\prime}-\eta & 0 & w\end{array}\right)$. 


$$
\begin{aligned}
& \sigma_{11}:=f+2(\delta a \varphi+\delta b \psi)-4 \eta^{\prime}, \quad \sigma_{12}:=-4 \delta \varphi-2 \eta \psi+4 \delta a^{\prime}, \quad \sigma_{13}:=4 \eta, \quad \sigma_{14}:=-4 \delta a, \\
& \sigma_{21}:=-4 \delta \psi+2 \varphi\left(w^{\prime}-\eta\right)+4 \delta b^{\prime}, \quad \sigma_{22}:=p, \quad \sigma_{23}:=-4 \delta b, \quad \sigma_{24}:=-4 \eta+4 w^{\prime}, \\
& \sigma_{31}:=-4 a \delta a-2 \delta \varphi \varphi+2 \delta \psi \psi-\eta \varphi \psi+f^{\prime}+4 \varphi \delta a^{\prime}-4 \delta a \varphi^{\prime}-2 \delta b \psi^{\prime}+\varphi \psi w^{\prime}-4 \eta_{x}, \\
& \sigma_{32}:=-2 \psi \eta^{\prime}-2 \psi \eta^{\prime}-2 \psi \varphi \delta a-4 \delta \varphi^{\prime}+2 \eta \psi^{\prime}+2 b w^{\prime}+2 \delta a_{x}, \\
& \sigma_{33}:=f, \quad \sigma_{34}:=-4 \delta \varphi-2 \psi\left(w^{\prime}-\eta\right), \\
& \sigma_{41}:=2 \delta b \varphi \psi+2 \varphi \eta^{\prime}+2 \varphi \eta^{\prime}-4 \delta \psi^{\prime}+2 \varphi^{\prime}\left(w^{\prime}-\eta\right)+2 a w^{\prime}+2 \delta b_{x}, \\
& \sigma_{42}:=-4 b \delta b+2 \delta \varphi \varphi-2 \delta \psi \psi-\eta \varphi \psi-2 \varphi \delta a^{\prime}+2 \psi \delta b^{\prime}+p^{\prime}-2 \delta b \psi^{\prime}, \\
& \sigma_{43}:=-4 \delta \psi+2 \eta \varphi, \quad \sigma_{44}:=2 \delta a \varphi+p+2 \delta b \psi-4 \eta^{\prime} .
\end{aligned}
$$

The odd superfield $\eta$ and the even superfields $w, f$ and $p$ are given by:

$$
\begin{aligned}
\eta_{x}= & b \delta b-\left(a+\varphi^{\prime}\right) \delta a+\varphi \delta a^{\prime}+\psi \delta \psi-\varphi \delta \varphi, \quad w_{x}=-\psi \delta b-\varphi \delta a, \\
f_{x}= & 2\left(2 b^{\prime}+\psi_{x}\right) \delta b-2 \psi \delta b_{x}+4\left(b+\psi^{\prime}\right) \delta \psi-4\left(a+\varphi^{\prime}\right) \delta \varphi+4 \varphi \delta \varphi^{\prime} \\
& -4\left(b \varphi+a \psi+\psi \varphi^{\prime}\right)\left(w^{\prime}-\eta\right)+4 \varphi \psi \eta^{\prime} . \\
p_{x}= & 2\left(2 b^{\prime}+\psi_{x}\right) \delta b+4 b \delta b^{\prime}+2 \psi \delta b_{x}-4 a \delta a^{\prime}-4 \varphi \delta a_{x}-4 b \delta \psi+4 a \delta \varphi+4 \varphi \delta \varphi^{\prime} \\
& -4\left(b \varphi+a \psi+\psi^{\prime} \varphi\right) \eta+4 \varphi \psi \eta^{\prime} .
\end{aligned}
$$

In particular:

$$
\begin{aligned}
& \mathscr{C}_{-1}^{\lambda}(m)=\left(\begin{array}{cccc}
-1 & 0 & 0 & 0 \\
0 & 1 & 0 & 0 \\
0 & 0 & -1 & 0 \\
0 & 0 & 0 & 1
\end{array}\right) \\
& \mathscr{C}_{0}^{\lambda}(m)=\frac{1}{2}\left(\begin{array}{cccc}
\psi \varphi+2 \lambda & 2 b & 0 & 2 \psi \\
2 a & \varphi \psi-2 \lambda & 2 \varphi & 0 \\
b \varphi+a \psi+\varphi \psi^{\prime} & 2 b^{\prime}+\psi_{x} & \psi \varphi+2 \lambda & 2 b+2 \psi^{\prime} \\
2 a^{\prime}+\varphi_{x} & b \varphi+a \psi+\psi \varphi^{\prime} & 2 a+2 \varphi^{\prime} & \varphi \psi-2 \lambda
\end{array}\right), \\
& \mathscr{C}_{1}^{\lambda}(m)=\frac{1}{4}\left(\begin{array}{llll}
c_{11} & c_{12} & c_{13} & c_{14} \\
c_{21} & c_{22} & c_{23} & c_{24} \\
c_{31} & c_{32} & c_{33} & c_{34} \\
c_{41} & c_{42} & c_{43} & c_{44}
\end{array}\right) \\
& c_{11}:=2 a b+2 \psi a^{\prime}-\varphi \psi_{x}-4 \lambda^{2} \text {, } \\
& c_{12}:=b \psi \varphi+2 b_{x}-4 \lambda b \text {, } \\
& c_{13}:=2 b \varphi+2 a \psi+2 \psi \varphi^{\prime} \text {, } \\
& c_{14}:=2 \psi_{x}-4 \lambda \psi \text {, } \\
& c_{21}:=a \psi \varphi-2 a_{x}-4 \lambda a, \\
& c_{22}:=-2 a b-2 \varphi b^{\prime}+\psi \varphi_{x}+4 \lambda^{2} \text {, } \\
& c_{23}:=-2 \varphi_{x}-4 \lambda \varphi \text {, } \\
& c_{24}:=-2 b \varphi-2 a \psi-2 \varphi \psi^{\prime} \text {, } \\
& c_{31}:=2 b a^{\prime}+2 a b^{\prime}+2 a^{\prime} \psi^{\prime}-\psi a_{x}+\varphi b_{x}+2 a \psi_{x}+\varphi \psi_{x}^{\prime}, \\
& c_{32}:=2 \psi \varphi b^{\prime}-b \psi \varphi^{\prime}-a \psi \psi^{\prime}-\psi \varphi^{\prime} \psi^{\prime}-\varphi \psi \psi_{x}+2 b_{x}^{\prime}+\psi_{x x}-4 \lambda b^{\prime}-2 \lambda \psi_{x} \text {, } \\
& c_{33}:=2 a b-2 \varphi b^{\prime}+2 b \varphi^{\prime}+2 a \psi^{\prime}+2 \varphi^{\prime} \psi^{\prime}-\psi \varphi_{x}-2 \varphi \psi_{x}-4 \lambda^{2} \text {, } \\
& c_{34}:=b \psi \varphi-\varphi \psi \psi^{\prime}+2 b_{x}+2 \psi_{x}^{\prime}-4 \lambda b-4 \lambda \psi^{\prime} \text {, } \\
& c_{41}:=2 \psi \varphi a^{\prime}+b \varphi \varphi^{\prime}+a \varphi \psi^{\prime}+\varphi \varphi^{\prime} \psi^{\prime}-\varphi \psi \varphi_{x}-2 a_{x}^{\prime}-\varphi_{x x}-4 \lambda a^{\prime}-2 \lambda \varphi_{x} \text {, } \\
& c_{42}:=-2 b a^{\prime}-2 a b^{\prime}-2 b^{\prime} \varphi^{\prime}-\psi a_{x}+\varphi b_{x}-2 b \varphi_{x}-\psi \varphi_{x}^{\prime} \text {, } \\
& c_{43}:=a \psi \varphi-\varphi \psi \varphi^{\prime}-2 a_{x}-2 \varphi_{x}^{\prime}-4 \lambda a-4 \lambda \varphi^{\prime}, \\
& c_{44}:=-2 a b+2 \psi a^{\prime}-2 b \varphi^{\prime}-2 a \psi^{\prime}-2 \varphi^{\prime} \psi^{\prime}+2 \psi \varphi_{x}+\varphi \psi_{x}+4 \lambda^{2} \text {. }
\end{aligned}
$$


Parameter independent Lax formulation: $d L(m) / d t_{j}=\left[L(m), C_{j}(m)\right](j=-1,0,1, \ldots)$ :

$$
\begin{aligned}
& L(m)=\left(\begin{array}{cc}
-\partial_{x} & -b-\psi D \\
a+\varphi D & \partial_{x}
\end{array}\right), \\
& C_{j}(m)=H\left(m, d_{j}(m)\right)+\sum_{k=0}^{j}\left(H\left(m, d_{J-k}(m)\right)+G\left(d_{j+1-k}(m)\right)\right) L^{k}(m) \\
& +(-1)^{j+1}\left(\begin{array}{cc}
-1 & 0 \\
0 & 1
\end{array}\right) L^{j+1}(m) \\
& H(m, \delta m)=\frac{1}{8}\left(\begin{array}{ll}
2 \sigma_{11}+\varphi \sigma_{14}+2 \sigma_{13} D & 2 \sigma_{12}+\psi \sigma_{13}+2 \sigma_{14} D \\
2 \sigma_{21}+\varphi \sigma_{24}+2 \sigma_{23} D & 2 \sigma_{22}+\psi \sigma_{23}+2 \sigma_{24} D
\end{array}\right), \quad G(m, \delta m)=\left(\begin{array}{cc}
4 w & 0 \\
0 & 4 w
\end{array}\right)
\end{aligned}
$$

( $w$ and $\sigma_{r s}$ are defined as functions of $m, \delta m$ in the first page of this table). In particular:

$$
\begin{aligned}
& C_{-1}(m)=\left(\begin{array}{cc}
-1 & 0 \\
0 & 1
\end{array}\right), \quad C_{0}(m)=\left(\begin{array}{cc}
-\partial_{x} & 0 \\
0 & -\partial_{x}
\end{array}\right), \quad C_{1}(m)=\frac{1}{2}\left(\begin{array}{ll}
c_{11} & c_{12} \\
c_{21} & c_{22}
\end{array}\right), \\
& c_{11}:=-2 \partial_{x x}+\varphi \psi \partial_{x}+\left(b \varphi+a \psi+\psi \varphi^{\prime}\right) D+a b+\psi a^{\prime}, \\
& c_{12}:=-2 \psi D \partial_{x}-2 b \partial_{x}-\psi_{x} D-b_{x}, \\
& c_{21}:=2 \varphi D \partial_{x}+2 a \partial_{x}+\varphi_{x} D+a_{x}, \\
& c_{22}:=2 \partial_{x x}+\varphi \psi \partial_{x}-\left(b \varphi+a \psi+\varphi \psi^{\prime}\right) D-a b-\varphi b^{\prime} .
\end{aligned}
$$

\section{Reductions of the susy AKNS}

We briefly recall some notions about the reduction of different tensor objects, that we are using in this section. Let $\mathscr{M}$ be an ordinary manifold or supermanifold and $\mathscr{M}^{\prime}$ a submanifold.

It is obvious that every function $h$ on $\mathscr{M}$ can be restricted to $\mathscr{M}^{\prime}$. A vector field $K$ on $\mathscr{M}$ can be restricted to $\mathscr{M}^{\prime}$ if $K(m) \in T_{m} \mathscr{M}^{\prime}$ for each $m \in \mathscr{M}^{\prime}$ (here, we use the natural inclusion $\left.T_{m} \mathscr{M}^{\prime} \subset T_{m} \mathscr{M}\right)$. In this case, $K$ gives rise to a vector field $K^{\prime}$ on $\mathscr{M}^{\prime}$.

A $(2,0)$ tensor $P$ on $\mathscr{M}$ can be restricted if the following two conditions are satisfied for each $m \in \mathscr{M}^{\prime}$ and each covector $\delta m^{\prime} \in T_{m}^{*} \mathscr{M}^{\prime}$ :

a) there is at least one covector $\delta m \in T_{m}^{*} \mathscr{M}$ extending $\delta m^{\prime}\left(\left\langle\delta m, \dot{m}^{\prime}\right\rangle=\left\langle\delta m^{\prime}, \dot{m}^{\prime}\right\rangle\right.$ for each $\dot{m}^{\prime} \in T_{m} \mathscr{M}^{\prime}$ ) and such that $P_{m} \delta m \in T_{m} \mathscr{M}^{\prime}$;

b) the vector $P_{m} \delta m \in T_{m} \mathscr{M}^{t}$ depends only on $\delta m^{\prime}$ and not on the particular extension $\delta m$ considered.

Under the above two conditions, there is a unique $(2,0)$ tensor $P^{\prime}$ on $\mathscr{M}^{\prime}$ such that $P_{m}^{\prime} \delta m^{\prime}=P_{m} \delta m$ for each $\delta m^{\prime}$ and each extension $\delta m$ as in (a). $P^{\prime}$ is called the restriction of $P$ to $\mathscr{M}^{\prime}$; the term "Dirac reduction" is also used in the literature.

A $(0,2)$ tensor $\Omega$ on $\mathscr{M}$ can always be restricted to a $(0,2)$ tensor $\Omega^{\prime}$ on $\mathscr{M}^{\prime}$; the latter is defined by $\left\langle\Omega_{m}^{\prime} \dot{m}^{\prime}, \dot{m}^{\prime \prime}\right\rangle:=\left\langle\Omega_{m} \dot{m}^{\prime}, \dot{m}^{\prime \prime}\right\rangle$ for each $m \in \mathscr{M}^{\prime}$ and $\dot{m}^{\prime}, \dot{m}^{\prime \prime} \in T_{m} \mathscr{M}^{\prime}$.

Finally, a $(1,1)$ tensor $N$ on $\mathscr{M}$ can be restricted if $N_{m}\left(T_{m} \mathscr{M}^{\prime}\right) \subset T_{m} \mathscr{M}^{\prime}$ for each $m \in \mathscr{M}^{\prime}$. If this condition is satisfied, we can define on $\mathscr{M}^{\prime}$ the restriction $N^{\prime}$, given by $N_{m}^{\prime} \dot{m}^{\prime}:=N_{m} \dot{m}^{\prime}$ for each $m \in \mathscr{M}^{\prime}, \dot{m}^{\prime} \in T_{m} \mathscr{M}^{\prime}$.

The restriction operation preserves:

i) the conserved quantities and the commutativity of vector fields;

ii) the Poisson property and the (pre)symplectic character for, respectively, $(2,0)$ and $(0,2)$ tensors; 
iii) the vanishing of the Nijenhuis torsion for $(1,1)$ tensors;

iv) the compatibility for a pair $Q, P$ of Poisson tensors, or a pair $\Omega, N$ formed by a (pre)symplectic tensor and a recursion operator;

v) the Lax formulation for vector fields.

We briefly explain the meaning of statement v). Assume a vector field $K$ on $\mathscr{M}$ admits a Lax formulation with parameter as in Eq. (2.2.5), or, alternatively, a parameter independent formulation as in Eq. (2.2.8); the linear operators giving the Lax pair act on some conveniently chosen vector space. Futhermore, suppose that $K$ can be restricted to a vector field $K^{\prime}$ on some submanifold $\mathscr{M}^{\prime} \subset \mathscr{M}$. Then $K^{\prime}$ also admits a Lax formulation, which is obtained simply by evaluating the Lax pair of $K$ at the points of $\mathscr{M}^{\prime}$.

In the sequel of this section, $\mathscr{M}$ will be the phase space of the susy AKNS theory, introduced in Sect. 2. We will discuss the restrictions of the theory to some significant submanifolds.

\subsection{The classical reduction. Let us consider the submanifold}

$\mathscr{M}_{\mathrm{cl}}:=\{(b, a, \psi, \varphi) \in \mathscr{M} \mid b=0, a=0, \psi(x, \theta)=\theta q(x), \varphi(x, \theta)=\theta r(x)\}$,

$q$ and $r$ being arbitrary even functions of the $x$ variable. $\mathscr{M}_{\mathrm{cl}}$ can be identified with a space of pairs $(q, r)$. For $m=(q, r) \in \mathscr{M}_{\mathrm{cl}}$, we represent a tangent vector and a covector at $m$ as pairs $\dot{m}=(\dot{q}, \dot{r})$ and $\delta m=(\delta q, \delta r)$, where all the components are even functions of $x$. We have the pairing $\langle\delta m, \dot{m}\rangle=\int d x(\delta q \dot{q}+\delta r \dot{r})$.

The Poisson tensors $Q, P$, the recursion operator $N$ and the hierarchy $h_{j}, K_{j}$ of Sect. 2 can be restricted to $\mathscr{M}_{\mathrm{cl}}$, giving the classical AKNS theory; in particular, by restricting the Poisson tensors $Q$ and $P$ we find the biHamiltonian structure known from the literature [Ma2, MP2]. The restriction of the susy hierarchy of Subsect. 2.1 gives the classical AKNS hierarchy formed by the vector fields $K_{-1}(q, r)=\left(\begin{array}{c}2 q \\ -2 r\end{array}\right), K_{0}(q, r)=\left(\begin{array}{c}q_{x} \\ r_{x}\end{array}\right), K_{1}(q, r)=\frac{1}{2}\left(\begin{array}{c}q_{x x}-2 q^{2} r \\ -r_{x x}+2 q r^{2}\end{array}\right)$, etc. and by the Hamiltonians $h_{0}(q, r)=\int d x q r, h_{1}(q, r)=\frac{1}{2} \int d x q_{x} r, h_{2}(q, r)=-\frac{1}{4} \int d x\left(q_{x} r_{x}+q^{2} r^{2}\right)$, etc.

Let us now discuss the reduction of the susy Lax formalism of Subsect. 2.2. Of course, the reduced theory on $\mathscr{M}_{\mathrm{cl}}$ admits Lax formulations as in Eqs. (2.2.5), (2.2.8), (2.2.9) and (2.2.10), where $\mathscr{L}^{\lambda}, \mathscr{C}_{j}, L$ and $C_{j}$ are evaluated at points $m=(0,0, \theta q, \theta r)$. We would like to recover from here the classical Lax formalism of the AKNS theory. To this purpose, we fix attention on Eq. (2.2.9); a straightforward computation shows that, at each point of $\mathscr{M}_{\mathrm{cl}}$, the operator $\left(\mathscr{L}^{\hat{\lambda}}\right)^{2}(m)$ leaves invariant the subspace $\mathscr{W}_{\mathrm{cl}} \subset \mathscr{W}$ formed by quadruples

$$
\Phi=\left(\begin{array}{c}
0 \\
0 \\
\theta f_{1} \\
\theta f_{2},
\end{array}\right)
$$

where $f_{1}=f_{1}(x)$ and $f_{2}=f_{2}(x)$ are even functions. We have

$$
\left(\mathscr{L}^{\lambda}\right)^{2}(m)\left(\begin{array}{c}
0 \\
0 \\
\theta f_{1} \\
\theta f_{2}
\end{array}\right)=\left(\begin{array}{c}
0 \\
0 \\
\theta g_{1} \\
\theta g_{2}
\end{array}\right),
$$


where

$$
\left(\begin{array}{l}
g_{1} \\
g_{2}
\end{array}\right)=\mathscr{L}_{\mathrm{cl}}^{\lambda}(m)\left(\begin{array}{l}
f_{1} \\
f_{2}
\end{array}\right), \quad \mathscr{L}_{\mathrm{cl}}^{\lambda}(m):=\left(\begin{array}{cc}
\partial_{x}+\lambda & q \\
r & \partial_{x}-\lambda
\end{array}\right) .
$$

The operator $\mathscr{L}_{\mathrm{cl}}^{\lambda}(m)$ appearing here acts on a space of pairs $\left(\begin{array}{l}f_{1}(x) \\ f_{2}(x)\end{array}\right)$, and is easily recognized as the Lax operator of the classical AKNS theory. By inspection, it is seen that the subspace $\mathscr{W}_{\mathrm{cl}}$ is invariant not only for $\left(\mathscr{L}^{\lambda}\right)^{2}(m)$, but also for the operators $\mathscr{C}_{j}^{\lambda}(m)$; so, working as in Eqs. (3.1.2), (3.1.3) we can construct from $\mathscr{C}_{j}^{\lambda}(m)$ an operator $C_{j, \mathrm{cl}}^{\lambda}(m)$ and we have a Lax formulation $d \mathscr{L}_{\mathrm{cl}}^{\lambda} / d t_{j}=\left[\mathscr{L}_{\mathrm{cl}}^{\lambda}, C_{j, \mathrm{cl}}^{\lambda}\right]$. For the lowest values of $j$, we find

$$
\begin{gathered}
C_{-1, \mathrm{cl}}^{\lambda}(m)=\left(\begin{array}{cc}
-1 & 0 \\
0 & 1
\end{array}\right), \quad C_{0, \mathrm{cl}}^{\lambda}(m)=\left(\begin{array}{cc}
\lambda & q \\
r & -\lambda
\end{array}\right), \\
C_{1, \mathrm{cl}}^{\lambda}(m)=\frac{1}{2}\left(\begin{array}{cc}
q r-2 \lambda^{2} & q_{x}-2 \lambda q \\
-r_{x}-2 \lambda r & -q r+2 \lambda^{2}
\end{array}\right), \\
C_{2, \mathrm{cl}}^{\lambda}(m)=\frac{1}{4}\left(\begin{array}{cc}
r q_{x}-q r_{x}-2 \lambda q r+4 \lambda^{3} & q_{x x}-2 q^{2} r-2 \lambda q_{x}+4 \lambda^{2} q \\
r_{x x}-2 q r^{2}+2 \lambda r_{x}+4 \lambda^{2} r & q r_{x}-r q_{x}+2 \lambda q r-4 \lambda^{3}
\end{array}\right) .
\end{gathered}
$$

These operators are known in the framework of the classical AKNS theory.

3.2. The susy nonlinear Schrödinger theory. Let us consider the submanifold

$$
\mathscr{M}_{0}:=\{(b, a, \psi, \varphi) \in \mathscr{M} \mid b=0, a=0\} .
$$

$\mathscr{M}_{0}$ can be identified with a space of pairs $(\psi, \varphi)$, where both superfields are odd.

The Poisson tensors $Q, P$, the recursion operator $N$ and all vector fields of the susy AKNS theory can be restricted to $\mathscr{M}_{0}$. It is easy to obtain the explicit expressions for the restrictions of the recursion operator and the symplectic tensor $\Omega=Q^{-1}$; they are reported in Table $2 \mathrm{a}$, together with the first vector fields and Hamiltonian functions; for simplicity, in the Table and in the sequel the restrictions of $\Omega, N, K_{j}$, etc. are denoted again by the same symbols.

The reduced theory admits Lax formulations in terms of $4 \times 4$ or $2 \times 2$ matrix superdifferential operators, which are obtained evaluating the operators of Sect. 2 at the points of $\mathscr{M}_{0}$; in particular, the associated spectral problem is as in Eq. (1.5) with $b=a=0$.

By performing a further reduction one obtains a theory in one odd superfield $\psi$, which can be viewed as a susy extension of the classical nonlinear Schrödinger (NLS) theory. Consider the submanifold $\mathscr{M}_{1} \subset \mathscr{M}_{0}$ formed by pairs $(\psi, \bar{\psi})$ (where the bar denotes complex conjugation as in Subsect. 1.1). Any such pair is uniquely determined by the first component, so we can identify $\mathscr{M}_{1}$ with a set of odd superfields $\psi$. We represent a tangent vector as an odd superfield $\dot{\psi}$, and a covector as an even superfield $\delta \psi$; the pairing is $\langle\delta \psi, \dot{\psi}\rangle:=\int d x d \theta(\delta \psi \bar{\psi}+\overline{\delta \psi} \dot{\psi})$. We remark that $\langle$,$\rangle is a real bilinear map; more generally, all tensors on \mathscr{M}_{1}$ appearing in our constructions are real multilinear maps. ${ }^{1}$

1 This means that we are considering $L_{0}$-bilinear or multilinear maps, where $L_{0}$ is the set of "real" even scalars as in Subsect. 1.1. 
We consider the recursion operator $N$, the symplectic tensor $\Omega$ and the hierarchy appearing in Table $2 \mathrm{a}$. One checks that $i N$ can be restricted to $\mathscr{M}_{1}$, as well as the vector field $i K_{-1}$; this implies that all vector fields $i^{j} K_{j}(j=-1,0,1, \ldots)$ can be restricted.

Let us consider the restrictions of $-i N,-i \Omega,-i^{j} K_{j}$ and of the Hamiltonian functions $-i^{j} h_{j}$; for simplicity, in the sequel and in Table $2 \mathrm{~b}$ they will be denoted, respectively, by $N, \Omega, K_{j}$ and $h_{j}$. These objects give rise to a hierarchy, satisfying a recursion scheme: the explicit expressions for $N, \Omega$ and the first vector fields and Hamiltonians are given in the table. This theory is a susy extension of the ordinary NLS theory; the latter can be obtained putting the constraint $\psi(x, \theta)=\theta q(x)$. In particular, the evolution equation corresponding to the vector field $K_{1}$ of Table $2 \mathrm{~b}$ gives rise to the NLS equation $i d q / d t=\frac{1}{2} q_{x x}-q^{2} \bar{q}$. The Lax formalism for the susy NLS is obtained from the susy $(\psi, \varphi)$ theory putting $\varphi=\bar{\psi}$. (The formalism becomes closer to the classical NLS, if the spectral parameter is written as $\lambda=i \mu$, with $\mu$ real.)

Remark. As anticipated in the Introduction, the superextensions of the NLS equation were recently investigated by Roelofs and Kersten [RK] using the theory of coverings $[\mathrm{RH}]$, an analogue of the prolongation method of Estabrook and Wahlquist [EW]. The authors obtained two super equations, indicated as "case A" and "case B," extending the classical NLS. The complete integrability in both cases was inferred via the construction of an infinite-dimensional coverings algebra and an autoBäcklund transformation. The phase space considered in [RK] is a set of pairs $(q, \omega)$, where $q=q(x)$ is an even field, and $\omega=\omega(x)$ is odd. The NLS superequations, their symmetries and conserved quantities are written in terms of the variables $q, \omega$; moreover, a superfield $\Phi(x, \theta):=\omega(x)+\theta q(x)$ is introduced, and it is shown that both super NLS equations admit a susy formulation in terms of the superderivation $D_{\theta}:=\theta \partial_{x}+\frac{1}{2} \partial_{\theta}$. We have checked that the first vector fields and Hamiltonian functions of our formulation correspond to homologous objects constructed in [RK] for the case A. In particular, the evolution equation $d \psi / d t=K_{1}(\psi)$, with $K_{1}$ as in Table $2 \mathrm{~b}$, can be identified (up to some rescalings) with the case A super NLS equation, setting $\psi(x, \theta)=\sqrt{2} \Phi\left(x, \frac{\theta}{\sqrt{2}}\right)=\sqrt{2} \omega(x)+\theta q(x)$. The main difference with the results of $[\mathrm{RK}]$ is that we have a manifestly supersymmetric construction of the hierarchy, a biHamiltonian structure and an explicit Lax formulation.

Note added in proof. In order to update the list of references about the susy nonlinear Schrödinger equation, we mention a preprint of Popowicz [Pop], which appeared after submission of this paper. In this preprint, an extended supersymmetrization of the NLS equation is presented, admitting a Lax formulation; according to the author, this model is different from the one of Roelofs-Kersten.

Table 2a. The $(\psi, \varphi)$ susy theory

(Pre)symplectic tensor at point $m=(\psi, \varphi)$ :

$$
\begin{gathered}
\Omega_{m}: T_{m} \mathscr{M}_{0} \rightarrow T_{m}^{*} \mathscr{M}_{0}, \quad \Omega_{m}\left(\begin{array}{c}
\dot{\psi} \\
\dot{\varphi}
\end{array}\right)=\frac{1}{2}\left(\begin{array}{cc}
\Omega_{\psi \psi} & \Omega_{\psi \varphi} \\
\Omega_{\varphi \psi} & \Omega_{\varphi \varphi}
\end{array}\right)\left(\begin{array}{c}
\dot{\psi} \\
\dot{\varphi}
\end{array}\right), \\
\Omega_{\psi \psi}:=\varphi^{\prime} \partial_{x}^{-1} \varphi-\varphi \partial_{x}^{-1} \varphi^{\prime}, \quad \Omega_{\psi \varphi}:=D-\varphi^{\prime} \partial_{x}^{-1} \psi-\varphi \partial_{x}^{-1} \psi D, \\
\Omega_{\varphi \psi}:=-D+\psi^{\prime} \partial_{x}^{-1} \varphi+\psi \partial_{x}^{-1} \varphi D, \quad \Omega_{\varphi \varphi}:=-\psi^{\prime} \partial_{x}^{-1} \psi+\psi \partial_{x}^{-1} \psi^{\prime} .
\end{gathered}
$$


Recursion operator at a point $m$ :

$$
N_{m}: T_{m} \mathscr{M}_{0} \rightarrow T_{m} \mathscr{M}_{0}, \quad N_{m}\left(\begin{array}{c}
\dot{\psi} \\
\dot{\varphi}
\end{array}\right)=\frac{1}{2}\left(\begin{array}{cc}
N_{\psi \psi} & N_{\psi \varphi} \\
N_{\varphi \psi} & N_{\varphi \varphi}
\end{array}\right)\left(\begin{array}{c}
\dot{\psi} \\
\dot{\varphi}
\end{array}\right),
$$

$N_{\psi \psi}:=\partial_{x}-\varphi \psi-\psi^{\prime} \partial_{x}^{-1} \varphi^{\prime}-\psi D^{-1} \varphi^{\prime}-\psi_{x} \partial_{x}^{-1} \varphi$,

$N_{\psi \varphi}:=-\psi^{\prime} \partial_{x}^{-1} \psi D-\psi D^{-1} \psi^{\prime}+\psi_{x} \partial_{x}^{-1} \psi$,

$N_{\varphi \psi}:=\varphi^{\prime} \partial_{x}^{-1} \varphi D+\varphi D^{-1} \varphi^{\prime}-\varphi_{x} \partial_{x}^{-1} \varphi$,

$N_{\varphi \varphi}:=-\partial_{x}-\varphi \psi+\varphi^{\prime} \partial_{x}^{-1} \psi^{\prime}+\varphi D^{-1} \psi^{\prime}+\varphi_{x} \partial_{x}^{-1} \psi$.

Recursion schemes:

$d h_{j+1}=N^{*} d h_{j}(j=0,1,2, \ldots), K_{j+1}=N K_{j}$ and $d h_{j+1}=\Omega K_{j}(j=-1,0,1, \ldots)$.

First vector fields: $K_{-1}(m)=\left(\begin{array}{c}2 \psi \\ -2 \varphi\end{array}\right), \quad K_{0}(m)=\left(\begin{array}{c}\psi_{x} \\ \varphi_{x}\end{array}\right)$,

$K_{1}(m)=\frac{1}{2}\left(\begin{array}{c}\psi_{x x}+2 \psi \varphi_{x}-2 \psi \psi^{\prime} \varphi^{\prime} \\ -\varphi_{x x}+2 \psi \varphi \varphi_{x}+2 \varphi \varphi^{\prime} \psi^{\prime}\end{array}\right)$,

$K_{2}(m)=\frac{1}{4}\left(\begin{array}{c}\psi_{x x x}+3 \psi \varphi \psi_{x x}-3 \psi \varphi^{\prime} \psi_{x}^{\prime}-3 \psi_{x} \varphi^{\prime} \psi^{\prime} \\ \varphi_{x x x}-3 \psi \varphi \varphi_{x x}-3 \varphi \psi^{\prime} \varphi_{x}^{\prime}-3 \varphi_{x} \varphi^{\prime} \psi^{\prime}\end{array}\right)$.

First Hamiltonian functions: $h_{0}(m)=-\int d x d \theta \psi \varphi^{\prime}, h_{1}(m)=\frac{1}{2} \int d x d \theta \varphi_{x} \psi^{\prime}$,

$h_{2}(m)=\frac{1}{4} \int d x d \theta\left(\varphi \varphi^{\prime} \psi^{\prime 2}+\psi \varphi \psi^{\prime} \varphi_{x}+\psi_{x} \varphi_{x}^{\prime}\right)$.

Parameter dependent Lax formulation: $d \mathscr{L}^{\lambda}(m) / d t_{j}=\left[\mathscr{L}^{\lambda}(m), \mathscr{C}_{j}^{\lambda}(m)\right](j=-1,0,1, \ldots)$ :

$\mathscr{L}^{\hat{\lambda}}(m):=\left(\begin{array}{cccc}D & -\frac{1}{2} \psi & -1 & 0 \\ -\frac{1}{2} \varphi & D & 0 & -1 \\ \frac{1}{4} \varphi \psi-\lambda & -\frac{1}{2} \psi^{\prime} & -D & -\frac{1}{2} \psi \\ -\frac{1}{2} \varphi^{\prime} & -\frac{1}{4} \varphi \psi+\lambda & -\frac{1}{2} \varphi & -D\end{array}\right)$.

The operators $\mathscr{C}_{j}^{\lambda}(m)$ are obtained evaluating the homologous operators of Table $1 \mathrm{~b}$ with $b=a=0$.

Parameter independent Lax formulation: $d L(m) / d t_{j}=\left[L(m), C_{j}(m)\right](j=-1,0,1, \ldots)$ :

$L(m):=\left(\begin{array}{cc}-\partial_{x} & -\psi D \\ \varphi D & \partial_{x}\end{array}\right)$.

The operators $C_{j}(m)$ are obtained evaluating the homologous operators of Table $1 \mathrm{~b}$ with $b=a=0$.

Table 2b. The susy NLS theory

(Pre)symplectic tensor at a point $\psi$ :

$$
\begin{gathered}
\Omega_{\psi}: T_{\psi} \mathscr{M}_{1} \rightarrow T_{\psi}^{*} \mathscr{M}_{1}, \quad \Omega_{\psi} \dot{\psi}=-\frac{i}{2} \Omega_{\psi \psi} \dot{\psi}-\frac{i}{2} \Omega_{\psi \bar{\psi}} \bar{\psi}, \\
\Omega_{\psi \psi}:=\overline{\psi^{\prime}} \partial_{x}^{-1} \bar{\psi}-\bar{\psi} \partial_{x}^{-1} \overline{\psi^{\prime}}, \quad \Omega_{\psi \bar{\psi}}:=D-\overline{\psi^{\prime}} \partial_{x}^{-1} \psi-\bar{\psi} \partial_{x}^{-1} \psi D .
\end{gathered}
$$

Recursion operator and its adjoint at a point $\psi$ :

$$
\begin{gathered}
N_{\psi}: T_{\psi} \mathscr{M}_{1} \rightarrow T_{\psi} \mathscr{M}_{1}, \quad N_{\psi} \dot{\psi}=-\frac{i}{2} N_{\psi \psi} \dot{\psi}-\frac{i}{2} N_{\psi \bar{\psi}} \overline{\dot{\psi}}, \\
N_{\psi \psi}:=\partial_{x}-\bar{\psi} \psi-\psi^{\prime} \partial_{x}^{-1} \overline{\psi^{\prime}}-\psi D^{-1} \overline{\psi^{\prime}}-\psi_{x} \partial_{x}^{-1} \bar{\psi}, \\
N_{\psi \bar{\psi}}:=-\psi^{\prime} \partial_{x}^{-1} \psi D-\psi D^{-1} \psi^{\prime}+\psi_{x} \partial_{x}^{-1} \psi, \\
N_{\psi}^{*}: T_{\psi}^{*} \mathscr{M}_{1} \rightarrow T_{\psi}^{*} \mathscr{M}_{1}, \quad N_{\psi}^{*} \delta \psi=-\frac{i}{2} N_{\psi \psi}^{*} \delta \psi-\frac{i}{2} N_{\psi \bar{\psi}}^{*} \overline{\delta \psi}, \\
N_{\psi \psi}^{*}:=-\partial_{x}+\bar{\psi} \psi+\psi^{\prime} \partial_{x}^{-1}\left(2 \overline{\psi^{\prime}}-\bar{\psi} D\right)-\bar{\psi} \partial_{x}^{-1} \bar{\psi}_{x}, \\
N_{\psi \bar{\psi}}^{*}:=-\psi^{\prime} \partial_{x}^{-1}\left(2 \psi^{\prime}-\psi D\right)+\psi \partial_{x}^{-1} \psi^{\prime} D .
\end{gathered}
$$


Recursion schemes:

$d h_{j+1}=N^{*} d h_{j}(j=0,1,2, \ldots), K_{j+1}=N K_{j}$ and $d h_{j+1}=\Omega K_{j}(j=-1,0,1, \ldots)$.

First vector fields: $K_{-1}(\psi)=2 i \psi, K_{0}(\psi)=\psi_{x}$,

$K_{1}(\psi)=-\frac{i}{2}\left(\psi_{x x}+2 \psi \bar{\psi} \psi_{x}-2 \psi \psi^{\prime} \overline{\psi^{\prime}}\right)$,

$K_{2}(\psi)=-\frac{1}{4}\left(\psi_{x x x}+3 \psi \bar{\psi} \psi_{x x}-3 \psi \overline{\psi^{\prime}} \psi_{x}^{\prime}-3 \psi_{x} \overline{\psi^{\prime}} \psi^{\prime}\right)$.

First Hamiltonian functions: $h_{0}(\psi)=-\int d x d \theta \psi \overline{\psi^{\prime}}, \quad h_{1}(\psi)=-\frac{i}{2} \int d x d \theta \overline{\psi_{x}} \psi^{\prime}$, $h_{2}(\psi)=-\frac{1}{4} \int d x d \theta\left(\bar{\psi} \psi^{\prime} \psi^{\prime 2}+\psi \bar{\psi} \psi^{\prime} \overline{\psi_{x}}+\psi_{x} \overline{\psi_{x}^{\prime}}\right)$.

Parameter dependent Lax formulation: $d \mathscr{L}^{\mu}(m) / d t_{j}=\left[\mathscr{L}^{\mu}(m),(-i)^{\mu} \mathscr{C}_{j}^{\mu}(m)\right](j=-1,0,1, \ldots)$

$$
\mathscr{L}^{\mu}(m):=\left(\begin{array}{cccc}
D & -\frac{1}{2} \psi & -1 & 0 \\
-\frac{1}{2} \bar{\psi} & D & 0 & -1 \\
\frac{1}{4} \bar{\psi} \psi-i \mu & -\frac{1}{2} \psi^{\prime} & -D & -\frac{1}{2} \psi \\
-\frac{1}{2} \bar{\psi}^{\prime} & -\frac{1}{4} \bar{\psi} \psi+i \mu & -\frac{1}{2} \bar{\psi} & -D
\end{array}\right) .
$$

The operators $\mathscr{C}_{j}^{\mu}(m)$ are obtained evaluating the operators $\mathscr{C}_{j}^{\lambda}(m)$ of Table $1 \mathrm{~b}$ with $b=a=0, \varphi=\bar{\psi}, \lambda=i \mu$.

Parameter independent Lax formulation: $\quad d L(m) / d t_{j}=\left[L(m),(-i)^{j} C_{J}(m)\right](j=-1,0,1 \ldots)$ $L(m):=\left(\begin{array}{cc}-\partial_{x} & -\psi D \\ \bar{\psi} D & \partial_{x}\end{array}\right)$.

The operators $C_{j}(m)$ are obtained evaluating the homologous operators of Table $1 \mathrm{~b}$ with $b=a=0, \varphi=\bar{\psi}$.

3.3. A susy modified KdV theory. Let us consider the submanifold

$$
\mathscr{M}_{2}:=\{(b, a, \psi, \varphi) \in \mathscr{M} \mid a=b, \varphi=\psi\} .
$$

This phase space can be identified with a set of pairs $(b, \psi)$. The square $J:=N^{2}$ of the recursion operator in Table $1 \mathrm{a}$ and the even order vector fields $K_{2 j}$ of the susy AKNS hierarchy can be restricted to $\mathscr{M}_{2}$. Also, we can restrict to this submanifold the symplectic tensor $\Omega$ of Table 1a, but the restrictions of the vector fields $K_{2 j}$ fail to be Hamiltonian with respect to the reduced tensor. In order to recover a Hamiltonian scheme on $\mathscr{M}_{2}$, we consider the $(0,2)$ type tensor $\Theta:=\Omega \circ N$ (which is a (pre)symplectic tensor on the susy AKNS phase space). The restrictions of $J, \Theta, K_{2 j}$ and $h_{2 j}$ to $\mathscr{M}_{2}$ will be denoted for simplicity with the same symbols. We can set up from these objects a biHamiltonian recursion scheme; the explicit expressions for $J, \Theta$ are reported in Table 3, together with the first vector fields and Hamiltonian functions. In this way, we obtain a susy $\mathrm{mKdV}$ (modified $\mathrm{KdV}$ ) theory. Indeed, it will be shown in the next subsection that this theory is related via a susy Miura transformation to the Manin-Radul susy KdV. Moreover, the classical $\mathrm{mKdV}$ theory can be obtained as a reduction of the susy $\mathrm{mKdV}$, corresponding to the constraints

$$
b=0, \quad \psi(x, \theta)=\theta v(x)
$$

( $v$ an arbitrary even function of $x$ ). Under these constraints, the evolution equation associated to the vector field $K_{2}$ becomes $d v / d t=\frac{1}{4} v_{x x x}-\frac{3}{2} v^{2} v_{x}$.

The susy mKdV hierarchy admits matrix Lax formulations as in Eqs. (2.2.5), (2.2.8), (2.2.9) and (2.2.10), where the involved linear operators are obtained evaluating the operators $\mathscr{L}^{\lambda}, \mathscr{C}_{2 j}^{\lambda}, L$ and $C_{2 j}$ of Table $1 \mathrm{~b}$ at $m=(b, b, \psi, \psi)$. There is 
also another Lax formulation in terms of scalar operators acting on a space of superfields $\chi=\chi(x, \theta)$. This formulation can be inferred from Eq. (2.2.10); in fact, for each $m \in \mathscr{M}_{2}$, the operator $L^{2}(m)$ leaves invariant the subspace $\mathscr{H}_{0} \subset \mathscr{H}$ formed by pairs $\Psi=\left(\begin{array}{l}\chi \\ \chi\end{array}\right)$ with equal components. It is found that

$$
\begin{aligned}
L^{2}(m)\left(\begin{array}{l}
\chi \\
\chi
\end{array}\right) & =\left(\begin{array}{c}
\Lambda(m) \chi \\
\Lambda(m) \chi
\end{array}\right), \\
\Lambda(m) & :=\partial_{x x}+\left(\psi_{x}-\psi \psi^{\prime}-2 b \psi\right) D+b_{x}-b^{2}+b^{\prime} \psi .
\end{aligned}
$$

By inspection, this subspace appears to be invariant also for the operators $C_{2 j}(m)$, so it is possible to define scalar operators $\Gamma_{2 j}(m)$ such that $C_{2 j}(m)\left(\begin{array}{l}\chi \\ \chi\end{array}\right)=\left(\begin{array}{l}\Gamma_{2 j}(m) \chi \\ \Gamma_{2 j}(m) \chi\end{array}\right)$. The first ones of these operators are reported in Table 3; they appear to be related to the fractional powers of $\Lambda$. (The existence of a scalar Lax formulation in terms of fractional powers could also be inferred a posteriori comparing the susy $\mathrm{mKdV}$ with the Manin-Radul susy KdV theory, which is known to admit such a Lax formulation; see the next subsection).

Table 3. The susy mKdV theory

(Pre)symplectic tensor at a point $m=(b, \psi)$ :

$$
\Theta_{m}: T_{m} \mathscr{M}_{2} \rightarrow T_{m}^{*} \mathscr{M}_{2}, \quad \Theta_{m}\left(\begin{array}{c}
\dot{b} \\
\dot{\psi}
\end{array}\right)=\frac{1}{2}\left(\begin{array}{cc}
\Theta_{b b} & \Theta_{b \psi} \\
\Theta_{\psi b} & \Theta_{\psi \psi}
\end{array}\right)\left(\begin{array}{c}
\dot{b} \\
\dot{\psi}
\end{array}\right),
$$

$$
\begin{aligned}
\Theta_{b b}:= & -4 \psi \partial_{x}^{-1}\left(2 b \psi+\psi \psi^{\prime}\right) \partial_{x}^{-1} \psi+2 \psi \partial_{x}^{-1}\left(\psi^{\prime}+2 b\right)+2\left(\psi^{\prime}+2 b\right) \partial_{x}^{-1} \psi, \\
\Theta_{b \psi}:= & -\partial_{x}-2 \psi \partial_{x}^{-1}\left(2 b \psi+\psi \psi^{\prime}\right) \partial_{x}^{-1}\left(2 b+\psi^{\prime}+\psi D\right)+2 \psi \partial_{x}^{-1}\left(b+\psi^{\prime}\right) D \\
& +\left(2 b+\psi^{\prime}\right) \partial_{x}^{-1}\left(2 b+\psi^{\prime}+\psi D\right), \\
\Theta_{\psi b}:= & \partial_{x}+4\left(b+\psi^{\prime}\right) D^{-1} \psi-4\left(b+\psi^{\prime}\right) \partial_{x}^{-1}\left(2 b \psi+\psi \psi^{\prime}\right) \partial_{x}^{-1} \psi \\
& -2\left(b-\psi^{\prime}\right) \partial_{x}^{-1}(2 b+\psi D)+2\left(\psi_{x}+3 b^{\prime}\right) \partial_{x}^{-1} \psi-3 \psi D^{-1}\left(\psi^{\prime}+2 b\right) \\
& +2 \psi D^{-1}\left(2 b \psi+\psi \psi^{\prime}\right) \partial_{x}^{-1} \psi \\
\Theta_{\psi \psi}:= & -D \partial_{x}-2\left(b+\psi^{\prime}\right) \psi-2\left(b+\psi^{\prime}\right) \partial_{x}^{-1}\left(2 b \psi+\psi \psi^{\prime}\right) \partial_{x}^{-1}\left(2 b+\psi^{\prime}+\psi D\right) \\
& +2\left(b+\psi^{\prime}\right) \partial_{x}^{-1}\left(\psi_{x}+2 b^{\prime}+2 b D+2 \psi^{\prime} D\right)+2\left(b-\psi^{\prime}\right) \partial_{x}^{-1} b^{\prime} \\
& +\left(\psi_{x}+3 b^{\prime}\right) \partial_{x}^{-1}\left(2 b+\psi^{\prime}+\psi D\right)+\psi D^{-1}\left(\psi_{x}+3 b^{\prime}\right) \\
& +\psi D^{-1}\left(2 b \psi+\psi \psi^{\prime}\right) \partial_{x}^{-1}\left(2 b+\psi^{\prime}+\psi D\right) .
\end{aligned}
$$

Recursion operator at a point $m$ :

$$
\begin{aligned}
& J_{m}: T_{m} \mathscr{M}_{2} \rightarrow T_{m} \mathscr{M}_{2}, \quad J_{m}\left(\begin{array}{c}
\dot{b} \\
\dot{\psi}
\end{array}\right)=\frac{1}{4}\left(\begin{array}{cc}
J_{b b} & J_{b \psi} \\
J_{\psi b} & J_{\psi \psi}
\end{array}\right)\left(\begin{array}{c}
\dot{b} \\
\dot{\psi}
\end{array}\right), \\
& J_{b b}:= \partial_{x x}-2\left(2 b \psi+\psi \psi^{\prime}\right) D-4 b^{2}-4 \psi b^{\prime}-2 \psi \psi_{x}+4 \psi D^{-1} b b^{\prime} \partial_{x}^{-1} \psi-2 \psi D^{-1} b \psi^{\prime} D^{-1} \psi \\
&+\psi D^{-1}\left(2 b \psi+\psi \psi^{\prime}\right) D^{-1}\left(2 b+\psi^{\prime}\right)+\psi D^{-1} \psi_{x}^{\prime}-\psi_{x} D^{-1}\left(2 b+\psi^{\prime}\right)+2 b_{x}^{\prime} \partial_{x}^{-1} \psi \\
&-2 b^{\prime} D^{-1}\left(2 b \psi+\psi \psi^{\prime}\right) \partial_{x}^{-1} \psi+2 b_{x} D^{-1} \psi+4 b_{x} \partial_{x}^{-1}\left(2 b \psi+\psi \psi^{\prime}\right) \partial_{x}^{-1} \psi-2 b_{x} \partial_{x}^{-1}\left(2 b+\psi^{\prime}\right), \\
& J_{b \psi}:= 4 b b^{\prime}+2 b^{\prime} \psi^{\prime}-2 \psi b_{x}+2 \psi D^{-1} b b^{\prime} \partial_{x}^{-1}\left(2 b+\psi^{\prime}+\psi D\right)-2 \psi D^{-1} b \psi^{\prime} D^{-1}\left(b+\psi^{\prime}\right) \\
&-\psi D^{-1}\left(2 b \psi+\psi \psi^{\prime}\right) D^{-1} b^{\prime}+2 \psi D^{-1} b\left(b+\psi^{\prime}\right) \psi+\psi D^{-1} b_{x}^{\prime}-b^{\prime} D^{-1} \psi_{x} \\
&-b^{\prime} D^{-1}\left(2 b \psi+\psi \psi^{\prime}\right) \partial_{x}^{-1}\left(\psi^{\prime}+2 b+\psi D\right)+2 b_{x} \partial_{x}^{-1}\left(b^{\prime}+\psi_{x}\right) \\
&+2 b_{x} \partial_{x}^{-1}\left(2 b \psi+\psi \psi^{\prime}\right) \partial_{x}^{-1}\left(2 b+\psi^{\prime}+\psi D\right)+b_{x}^{\prime} \partial_{x}^{-1}\left(2 b+\psi^{\prime}+\psi D\right)+\psi_{x} D^{-1} b^{\prime}, \\
& J_{\psi b}:=-4\left(2 b \psi+\psi \psi^{\prime}\right)+2 \psi^{\prime} D^{-1}\left(2 b \psi+\psi \psi^{\prime}\right) \partial_{x}^{-1} \psi-4 b_{x} \partial_{x}^{-1} \psi-2 \psi_{x} \partial_{x}^{-1}\left(2 b+\psi^{\prime}\right) \\
&+4 \psi_{x} \partial_{x}^{-1}\left(2 b \psi+\psi \psi^{\prime}\right) \partial_{x}^{-1} \psi-2 \psi_{x}^{\prime} \partial_{x}^{-1} \psi,
\end{aligned}
$$




$$
\begin{aligned}
J_{\psi \psi}:= & \partial_{x x}-2 \psi\left(2 b+\psi^{\prime}\right) D-4 b\left(b+\psi^{\prime}\right)-2 \psi^{\prime 2}+\psi^{\prime} D^{-1}\left(2 b \psi+\psi \psi^{\prime}\right) \partial_{x}^{-1}\left(2 b+\psi^{\prime}+\psi D\right) \\
& +\psi^{\prime} D^{-1} \psi_{x}-2 b_{x} \partial_{x}^{-1}\left(2 b+\psi^{\prime}+\psi D\right)+2 \psi_{x} \partial_{x}^{-1}\left(2 b \psi+\psi \psi^{\prime}\right) \partial_{x}^{-1}\left(2 b+\psi^{\prime}+\psi D\right) \\
& -2 \psi_{x} \partial_{x}^{-1}\left(b+\psi^{\prime}\right) D-\psi_{x}^{\prime} \partial_{x}^{-1}\left(2 b+\psi^{\prime}+\psi D\right) .
\end{aligned}
$$

Recursion schemes: $d h_{2 J+2}=J^{*} d h_{2 j}=\Theta K_{2 J}$ and $K_{2 j+2}=J K_{2 j}(j=0,1,2, \ldots)$.

First vector fields: $K_{0}(m):=\left(\begin{array}{l}b_{x} \\ \psi_{x}\end{array}\right)$,

$K_{2}(m)=\frac{1}{4}\left(\begin{array}{c}b_{x x x}-3 b_{x} \psi \psi_{x}+3 b^{\prime} \psi^{\prime} \psi_{x}-3 \psi \psi^{\prime} b_{x}^{\prime}-6 b b_{x}^{\prime} \psi+6 b b^{\prime} \psi_{x}+6 b^{\prime} b_{x} \psi-6 b^{2} b_{x} \\ \psi_{x x x}-3 \psi \psi^{\prime} \psi_{x}^{\prime}-6 b \psi \psi_{x}^{\prime}-6 b \psi^{\prime} \psi_{x}-3 \psi^{\prime 2} \psi_{x}-6 b^{2} \psi_{x}-6 \psi \psi^{\prime} b_{x}-12 b b_{x} \psi\end{array}\right)$.

First Hamiltonian functions: $h_{0}(m)=-\int d x d \theta\left(2 b \psi+\psi \psi^{\prime}\right)$,

$h_{2}(m)=\frac{1}{4} \int d x d \theta\left(4 b^{3} \psi+6 b^{2} \psi \psi^{\prime}+4 b \psi \psi^{\prime 2}+\psi \psi^{\prime 3}+\psi_{x} \psi_{x}^{\prime}+2 b_{x} \psi_{x}\right)$.

Parameter dependent Lax formulation: $d \mathscr{L}^{\hat{\lambda}}(m) / d t_{2 j}=\left[\mathscr{L}^{\lambda}(m), \mathscr{C}_{2 j}^{\lambda}(m)\right](j=0,1,2, \ldots)$ :

$\mathscr{L}^{\lambda}(m):=\left(\begin{array}{cccc}D & -\frac{1}{2} \psi & -1 & 0 \\ -\frac{1}{2} \psi & D & 0 & -1 \\ -\lambda & -b-\frac{1}{2} \psi^{\prime} & -D & -\frac{1}{2} \psi \\ -b-\frac{1}{2} \psi^{\prime} & \lambda & -\frac{1}{2} \psi & -D\end{array}\right)$.

The operators $\mathscr{C}_{2 j}(m)$ are obtained evaluating the homologous operators of Table $1 \mathrm{~b}$ with $a=b, \varphi=\psi$.

Parameter independent Lax formulation: $d L(m) / d t_{2 j}=\left[L(m), C_{2 j}(m)\right] \quad(j=0,1,2, \ldots)$ :

$L(m):=\left(\begin{array}{cc}-\partial_{x} & -b-\psi D \\ b+\psi D & \partial_{x}\end{array}\right)$.

The operators $C_{2 j}(m)$ are obtained evaluating the homologous operators of Table $1 \mathrm{~b}$ with $a=b, \varphi=\psi$.

Scalar Lax formulation: $d \Lambda(m) / d t_{2 j}=\left[\Lambda(m), \Gamma_{2 j}(m)\right] \quad(j=0,1,2, \ldots)$ :

$\Lambda(m):=\partial_{x x}+\left(\psi_{x}-\psi \psi^{\prime}-2 b \psi\right) D+b_{x}-b^{2}+b^{\prime} \psi$,

$\Gamma_{0}(m):=-\partial_{x}=-\left(\Lambda^{\frac{1}{2}}\right)_{+}(m)$,

$\Gamma_{2}(m):=-\partial_{x x x}+\frac{3}{2}\left(2 b \psi+\psi \psi^{\prime}-\psi_{x}\right) D \partial_{x}+\frac{3}{2}\left(b^{2}+\psi b^{\prime}-b_{x}\right) \partial_{x}$

$$
+\frac{3}{4}\left(2 \psi b+\psi^{\prime} \psi-\psi_{x}\right)_{x} D+\frac{3}{4}\left(b^{2}-b^{\prime} \psi-b_{x}\right)_{x}=-\left(\Lambda^{\frac{3}{2}}\right)_{+}(m) .
$$

$(+$ denotes projection on the purely differential part of scalar super pseudo-differential operators in D.)

3.4. The susy KdV theory of Manin-Radul. The Manin-Radul theory is well known from the literature [MR, OP, MP1]; here, we show that it can also be obtained from the susy AKNS by reduction to the submanifold

$$
\mathscr{M}_{3}:=\{(b, a, \psi, \varphi) \in \mathscr{M} \mid b=-1, \psi=0\} .
$$

This submanifold can be identified with a set of pairs $(a, \varphi)$. The square $J:=$ $N^{2}$ of the recursion operator in Table 1a and the even order vector fields $K_{2 j}$ of the susy AKNS hierarchy can be restricted to $\mathscr{M}_{3}$; the first Poisson tensor $Q$ of Table la cannot be restricted and its inverse $\Omega$ is reduced to zero on $\mathscr{M}_{3}$. For our purposes, it is convenient to consider the $(0,2)$ type tensor $\Theta:=\Omega \circ N$ (which is a (pre)symplectic tensor on the susy AKNS phase space). The restrictions of $\Theta, J$, $K_{2 j}$ and $h_{2 j}$ to $\mathscr{M}_{3}$ will be denoted for brevity with the same symbols. We can set up from these objects a recursion scheme; the explicit expressions for $\Theta$ and $J$ are reported in Table 4, together with the first vector fields and Hamiltonian functions. 
One recognizes the already known biHamiltonian framework of the Manin-Radul theory. ${ }^{2}$

The ordinary KdV theory can be obtained from the present one via the reduction

$$
a=0, \quad \varphi(x, \theta)=\theta u(x)
$$

( $u$ an arbitrary even function of $x$ ). In particular, under this constraint the evolution equation associated to the vector field $K_{2}$ becomes $d u / d t=\frac{1}{4} u_{x x x}+\frac{3}{2} u u_{x}$.

The Manin-Radul susy KdV admits matrix Lax formulations as in Eqs. (2.2.5), $(2.2 .8),(2.2 .9)$ and $(2.2 .10)$, where the involved linear operators are obtained evaluating the operators $\mathscr{L}^{\lambda}, \mathscr{C}_{2 j}^{\lambda}, L$ and $C_{2 j}$ of Table $1 \mathrm{~b}$ at $m=(-1, a, 0, \varphi)$. There is also another Lax formulation in terms of scalar operators acting on a space of superfields $\chi=\chi(x, \theta)$. This formulation can be inferred from Eq. (2.2.10); in fact, for each $m \in \mathscr{M}_{3}$, the operator $L^{2}(m)$ leaves invariant the subspace $\mathscr{H}_{0} \subset \mathscr{H}$ formed by pairs $\Psi=\left(\begin{array}{l}\chi \\ \chi_{x}\end{array}\right)$. It is found that

$$
L^{2}(m)\left(\begin{array}{c}
\chi \\
\chi_{x}
\end{array}\right)=\left(\begin{array}{c}
\Lambda(m) \chi \\
(\Lambda(m) \chi)_{x}
\end{array}\right), \quad \Lambda(m):=\partial_{x x}+\varphi D+a
$$

By inspection, $\mathscr{H}_{0}$ also appears to be invariant for the operators $C_{2 j}(m)$, so it is possible to define scalar operators $\Gamma_{2 j}(m)$ such that $C_{2 j}(m)\left(\begin{array}{c}\chi \\ \chi x\end{array}\right)=\left(\begin{array}{c}\Gamma_{2 j}(m) \chi \\ \left(\Gamma_{2 j}(m) \chi\right)_{x}\end{array}\right)$. The first ones of these operators are reported in Table 4 . They are related to the fractional powers of $\Lambda$; the scalar Lax formalism obtained in this way is familiar in the literature on the Manin-Radul theory.

We conclude discussing the connections between this theory and the susy mKdV of the previous subsection. By comparing the operators $\Lambda(m)$ in Eqs. (3.3.3) and (3.4.3) we are led to introduce the transformation $\mathscr{F}: \mathscr{M}_{2} \rightarrow \mathscr{M}_{3},(b, \psi) \mapsto(a, \varphi)$, where

$$
a=b_{x}-b^{2}+b^{\prime} \psi, \quad \varphi=\psi_{x}-\psi \psi^{\prime}-2 b \psi .
$$

The (pre)symplectic tensor $\Theta$ and the recursion operator $J$ of Table 3 are converted via $\mathscr{F}$ into the corresponding objects of Table 4 . Also, the vector fields and the Hamiltonian functions of the susy $\mathrm{mKdV}$ are transformed via $\mathscr{F}$ into the ones of the Manin-Radul theory. For this reason, we call $\mathscr{F}$ a susy Miura map. Under the constraints (3.3.2) and (3.4.2), we obtain from $\mathscr{F}$ the classical Miura map $u=v_{x}-v^{2}$.

The map (3.4.4) contains a supersymmetric transformation known from the literature. Indeed, (3.4.4) is compatible with the constraints $a=0, b=0$, and induces in this way the map $\psi \mapsto \varphi=\psi_{x}-\psi \psi^{\prime}$, appearing in [RH]. As it was shown in the quoted paper, this transformation relates a susy $\mathrm{mKdV}$ theory in one superfield $\psi$ with a susy $\mathrm{KdV}$ theory in $\varphi$. In the present framework, the $\psi$ theory can be obtained from Table 3 putting the constraint $b=0$; the $\varphi$ theory can be deduced from the Manin-Radul KdV of Table 4 putting $a=0$, and corresponds to the well known susy $\mathrm{KdV}$ of Mathieu [Mat].

2 In order to compare Table 4 with the description of the Manin-Radul theory given in our previous works [MP1, MP4], it is necessary to perform a reflection $\varphi \mapsto-\varphi$. In the quoted papers, we present a biHamiltonian scheme based on a pair of Poisson tensors $Q, P$; the pair $\Theta, J$ of Table 4 is related to $Q, P$ by $\Theta=2 Q^{-1}$ and $J=-P_{\circ} Q^{-1}$. 
Table 4. Manin-Radul theory

(Pre)symplectic tensor at a point $m=(a, \varphi)$ :

$$
\Theta_{m}: T_{m} \mathscr{M}_{3} \rightarrow T_{m}^{*} \mathscr{M}_{3}, \quad \Theta_{m}\left(\begin{array}{c}
\dot{a} \\
\dot{\varphi}
\end{array}\right)=\frac{1}{2}\left(\begin{array}{cc}
0 & \partial_{x}^{-1} \\
\partial_{x}^{-1} & D^{-1}+\partial_{x}^{-1} \varphi \partial_{x}^{-1}
\end{array}\right)\left(\begin{array}{c}
\dot{a} \\
\dot{\varphi}
\end{array}\right) .
$$

Recursion operator at a point $m$ :

$$
\begin{gathered}
J_{m}: T_{m} \mathscr{M}_{3} \rightarrow T_{m} \mathscr{M}_{3}, \quad J_{m}\left(\begin{array}{c}
\dot{a} \\
\dot{\varphi}
\end{array}\right)=\frac{1}{4}\left(\begin{array}{cc}
J_{a a} & J_{a \varphi} \\
J_{\varphi a} & J_{\varphi \varphi}
\end{array}\right)\left(\begin{array}{c}
\dot{a} \\
\dot{\varphi}
\end{array}\right), \\
J_{a a}:=\partial_{x x}+2 \varphi D+4 a+\varphi D^{-1} \varphi D^{-1}+2 a_{x} \partial_{x}^{-1}+\varphi_{x} D^{-1}, J_{\varphi a}:=4 \varphi+2 \varphi_{x} \partial_{x}^{-1}, \\
J_{a \varphi}:=-2 a^{\prime}+\left(a \varphi-a_{x}^{\prime}\right) \partial_{x}^{-1}-a^{\prime} D^{-1} \varphi \partial_{x}^{-1}-\varphi D^{-1} a D^{-1}+2 a_{x} \partial_{x}^{-1} \varphi \partial_{x}^{-1}, \\
J_{\varphi \varphi}:=\partial_{x x}+2 \varphi D+4 a+2 \varphi^{\prime}+\varphi^{\prime} D^{-1} \varphi \partial_{x}^{-1}+\left(2 a_{x}+\varphi_{x}^{\prime}\right) \partial_{x}^{-1}+\varphi_{x} \partial_{x}^{-1}\left(D+2 \varphi \partial_{x}^{-1}\right) .
\end{gathered}
$$

Recursion schemes: $d h_{2 j+2}=N^{*} d h_{2 j}=\Theta K_{2 j}$ and $K_{2 j+2}=J K_{j}(j=0,1,2, \ldots)$.

First vector fields: $K_{0}(m)=\left(\begin{array}{c}a_{x} \\ \varphi_{x}\end{array}\right), \quad K_{2}(m)=\frac{1}{4} \partial_{x}\left(\begin{array}{c}a_{x x}+3 a^{2}+3 \varphi a^{\prime} \\ \varphi_{x x}+3 \varphi \varphi^{\prime}+6 a \varphi\end{array}\right)$.

First Hamiltonian functions: $h_{0}(m)=\int d x d \theta \varphi, h_{2}(m)=\frac{1}{4} \int d x d \theta\left(\varphi \varphi^{\prime}+2 a \varphi\right)$.

Parameter dependent Lax formulation: $d \mathscr{L}^{\lambda}(m) / d t_{j}=\left[\mathscr{L}^{\lambda}(m), \mathscr{C}_{2 j}^{\lambda}(m)\right](j=0,1,2, \ldots)$ :

$$
\mathscr{L}^{\lambda}(m):=\left(\begin{array}{cccc}
D & 0 & -1 & 0 \\
-\frac{1}{2} \varphi & D & 0 & -1 \\
-\lambda & 1 & -D & 0 \\
-a-\frac{1}{2} \varphi^{\prime} & \lambda & 0 & -D
\end{array}\right)
$$

The operators $\mathscr{C}_{2 j}^{\lambda}(m)$ are obtained evaluating the homologous operators of Table $1 \mathrm{~b}$ with $b=-1$, $\psi=0$.

Parameter independent Lax formulation: $d L(m) / d t_{2 j}=\left[L(m), C_{2 j}(m)\right](j=0,1,2, \ldots)$ :

$L(m):=\left(\begin{array}{cc}-\partial_{x} & 1 \\ a+\varphi D & \partial_{x}\end{array}\right)$.

The operators $C_{2 j}(m)$ are obtained evaluating the homologous operators of Table $1 \mathrm{~b}$ with $b=-1, \psi=0$.

Scalar Lax formulation: $d \Lambda(m) / d t_{2 j}=\left[\Lambda(m), \Gamma_{2 j}(m)\right](j=0,1,2, \ldots)$ :

$\Lambda(m):=\partial_{x x}+\varphi D+a, \quad \Gamma_{0}(m):=-\partial_{x}=-\left(\Lambda^{\frac{1}{2}}\right)_{+}(m)$,

$\Gamma_{2}(m):=-\partial_{x x x}-\frac{3}{2} \varphi D \partial_{x}-\frac{3}{2} a \partial_{x}-\frac{3}{4} \varphi_{x} D-\frac{3}{4} a_{x}=-\left(\Lambda^{\frac{3}{2}}\right)_{+}(m)$.

\section{Description of the Method: The BiHamiltonian Reduction}

The method employed to construct the susy AKNS theory of Sect. 2 is strictly related to the general framework of [MP1-2]. The main points are:

i) the loop superalgebras and their biHamiltonian structures;

ii) a reduction theorem holding for general biHamiltonian manifolds [CMP, CP], which can also be used in the susy case and can be applied in particular to loop superalgebras.

The phase space of the susy AKNS theory is the quotient biHamiltonian manifold arising from the reduction of the loop superalgebra $\mathscr{G}$ associated to the Lie superalgebra $g l(2,2)$. As explained in Subsect. 1.4, the elements of $\mathscr{G}$ are $4 \times 4$ 
matrices, whose entries are superfields depending on $x$ and $\theta$; we have a grading $\mathscr{G}=\mathscr{G}_{0} \oplus \mathscr{G}_{1}$ and a structure of Lie superalgebra given by the supercommutator [, ].

We consider $\mathscr{G}_{1}$ as a supermanifold. At every point $V \in \mathscr{G}_{1}$, the tangent space is identified with $\mathscr{G}_{1}$ itself, and the cotangent space with $\mathscr{G}_{0}$. As usually, we typically denote tangent vectors and covectors with $\dot{V}$ and $\delta V$, respectively; the pairing is

$$
\langle\delta V, \dot{V}\rangle:=\int d x d \theta \operatorname{Tr}(\delta V \dot{V}) .
$$

$\mathscr{G}_{1}$ is a biHamiltonian supermanifold; the Poisson tensors $Q, P$ at a point $V$ are given by

$$
\begin{gathered}
Q_{V}: T_{V}^{*} \mathscr{G}_{1} \approx \mathscr{G}_{0} \rightarrow T_{V} \mathscr{G}_{1} \approx \mathscr{G}_{1}, \quad Q_{V}(\delta V):=[A, \delta V], \\
P_{V}: T_{V}^{*} \mathscr{G}_{1} \approx \mathscr{G}_{0} \rightarrow T_{V} \mathscr{G}_{1} \approx \mathscr{G}_{1}, \quad P_{V}(\delta V):=D(\delta V)+[V, \delta V] .
\end{gathered}
$$

Here, $A$ is an odd supermatrix with constant entries; for any choice of $A$, the above equations define a pair of compatible Poisson tensors. The choice giving rise to the susy AKNS theory is $A:=\left(\begin{array}{cccc}0 & 0 & 0 & 0 \\ 0 & 0 & 0 & 0 \\ -1 & 0 & 0 & 0 \\ 0 & 1 & 0 & 0\end{array}\right)$, as anticipated in the Introduction.

The biHamiltonian manifold $\left(\mathscr{G}_{1}, Q, P\right)$ can be reduced using the technique of [CMP, CP]. Essentially, one has to choose a symplectic leaf $\mathscr{S}$ of $Q$ (i.e., an integral leaf of the distribution $\operatorname{Im} Q) ; \mathscr{S}$ carries a structure of fibered space, the fibers being the integral leaves of the distribution $\mathscr{E}:=T \mathscr{S} \cap \mathscr{D}$, where $\mathscr{D}:=$ $P(\operatorname{Ker} Q)$. Both Poisson tensors $Q$ and $P$ can be reduced on the quotient space $\mathscr{M}:=\mathscr{S} / \mathscr{E}$, in an appropriate sense which is specified in the sequel. Let us summarize the main steps in this construction.

i) We choose the symplectic leaf $\mathscr{S}$ of $Q$ passing through the point $B=\left(\begin{array}{cccc}0 & 0 & -1 & 0 \\ 0 & 0 & 0 & -1 \\ 0 & 0 & 0 & 0 \\ 0 & 0 & 0 & 0\end{array}\right)$. It is found that

$$
\mathscr{S}:=\left\{S \in \mathscr{G}_{1} \mid S=\left(\begin{array}{cccc}
\mu & v & -1 & 0 \\
\sigma & \tau & 0 & -1 \\
d & g & -\mu & v \\
e & n & \sigma & -\tau
\end{array}\right)\right\},
$$

where $d(x, \theta), e(x, \theta), g(x, \theta), n(x, \theta)$ are even superfields, and $\mu(x, \theta), v(x, \theta)$, $\sigma(x, \theta), \tau(x, \theta)$ are odd superfields. As well as for $A$, the choice of $B$ is suggested by the form that we expect for the spectral problem of the susy AKNS (see the discussion in the following section).

ii) The distributions $\mathscr{D}$ and $\mathscr{E}$ at any point $S$ of $\mathscr{S}$ are given by

$$
\mathscr{D}_{S}=\left\{\dot{S} \in \mathscr{G}_{1} \mid \dot{S}=\left(\begin{array}{cccc}
q^{\prime}+p^{\prime}-\varepsilon & -v q-\rho-f^{\prime} & 0 & -2 f \\
+f \sigma-s \nu & +f(\tau-\mu) & & \\
\sigma q-\xi-s^{\prime} & p^{\prime}+\varepsilon-\eta & -2 s & 0 \\
+s(\mu-\tau) & +s v-f \sigma & & \\
-\varepsilon^{\prime}-e f-g s & v \eta-g q-\rho^{\prime} & \varepsilon-q^{\prime}-p^{\prime} & \rho-f^{\prime}-v q \\
+v \xi-\rho \sigma & +\rho(\mu-\tau) & +s v-f \sigma & +f(\tau-\mu) \\
& -f(d+n) & & \\
& & & \\
\sigma \eta+e q-\xi^{\prime} & \varepsilon^{\prime}-\eta^{\prime}+v \xi+\sigma \rho & \sigma q+\xi-s^{\prime} & \eta-\varepsilon-p^{\prime} \\
+\xi(\tau-\mu) & -g s-e f & +s(\mu-\tau) & +\sigma f-v s \\
-(d+n) s & & &
\end{array}\right\},\right.
$$




$$
\mathscr{E}_{S}=\left\{\dot{S} \in \mathscr{G}_{1} \mid \dot{S}=\left(\begin{array}{cccc}
q^{\prime}+p^{\prime}-\varepsilon & -v q & 0 & 0 \\
\sigma q & p^{\prime}+\varepsilon-\eta & 0 & 0 \\
-\varepsilon^{\prime} & v \eta-g q & \varepsilon-q^{\prime}-p^{\prime} & -v q \\
e q+\sigma \eta & \varepsilon^{\prime}-\eta^{\prime} & \sigma q & \eta-\varepsilon-p^{\prime}
\end{array}\right)\right\}
$$

where $p, q, f, s$ are arbitrary even superfields, and $\varepsilon, \eta, \rho, \xi$ are arbitrary and odd; these superfields play the role of arbitrary parameters.

A tangent vector to $\mathscr{S}$ can be written as $\dot{S}=\left(\begin{array}{cccc}\mu & v & 0 & 0 \\ \sigma & i & 0 & 0 \\ d & g & -\mu & v \\ e & n & \sigma & -i\end{array}\right)$; by comparing with Eq. (4.6), we find that $\dot{S} \in \mathscr{E}_{S}$ iff

$$
\begin{aligned}
\dot{v}+v\left(\partial_{x}^{-1}(\dot{n}-\dot{d})+D^{-1}(\dot{\mu}-\dot{\tau})\right) & =0, \\
\dot{\sigma}-\sigma\left(\partial_{x}^{-1}(\dot{n}-\dot{d})+D^{-1}(\dot{\mu}-\dot{\tau})\right) & =0, \\
\dot{g}+g\left(\partial_{x}^{-1}(\dot{n}-\dot{d})+D^{-1}(\dot{\mu}-\dot{\tau})\right)+v D^{-1}(\dot{n}+\dot{d}) & =0, \\
\dot{e}-e\left(\partial_{x}^{-1}(\dot{n}-\dot{d})+D^{-1}(\dot{\mu}-\dot{\tau})\right)+\sigma D^{-1}(\dot{n}+\dot{d}) & =0 .
\end{aligned}
$$

iii) We now integrate the distribution $\mathscr{E}$. It is found that the quotient space $\mathscr{M}=\mathscr{S} / \mathscr{E}$ can be identified with a space of quadruples $m=(b, a, \psi, \varphi)$, where $b, a$ are even superfields, and $\psi, \varphi$ are odd; the quotient map is $\pi: \mathscr{S} \rightarrow \mathscr{M}, S \mapsto$ $\pi(S)=(b, a, \psi, \varphi)$, where

$$
\begin{aligned}
b:=\frac{1}{2}\left(1-\beta^{\prime}-\beta f_{x}^{\prime}\right) \exp (-f), & a:=2\left(h-\gamma^{\prime}+\gamma f_{x}\right) \exp (f), \\
\psi:=\beta \exp (-f), \quad \varphi:=4 \gamma \exp (f), &
\end{aligned}
$$

and $\beta, \gamma, h, f$ are expressed as follows in terms of the elements of the matrix $S$ :

$$
\begin{aligned}
\beta=\frac{v}{g}, \quad \gamma & =\sigma g \exp \left(\left(\frac{\sigma}{e}+\frac{v}{g}\right) D^{-1}(n+d)\right), \\
h & =e g \exp \left(\left(\frac{\sigma}{e}+\frac{v}{g}\right) D^{-1}(n+d)\right), \\
f_{x} & =d-n+\tau^{\prime}-\mu^{\prime}-\frac{g_{x}}{g}-\left(\frac{v}{g} D^{-1}(n+d)\right)_{x} \\
& +2 v \sigma \exp \left(\left(\frac{\sigma}{e}+\frac{v}{g}\right) D^{-1}(n+d)\right) .
\end{aligned}
$$

For each $m \in \mathscr{M}$, the corresponding fiber $\pi^{-1}(m)$ contains an element of the form

$$
\Sigma(m)=\left(\begin{array}{cccc}
0 & -\frac{1}{2} \psi & -1 & 0 \\
-\frac{1}{2} \varphi & 0 & 0 & -1 \\
\frac{1}{4} \varphi \psi & -b-\frac{1}{2} \psi^{\prime} & 0 & -\frac{1}{2} \psi \\
-a-\frac{1}{2} \varphi^{\prime} & \frac{1}{4} \psi \varphi & -\frac{1}{2} \varphi & 0
\end{array}\right) .
$$

The map $m \mapsto \Sigma(m)$ is a cross section of the fibration $\mathscr{S} \rightarrow \mathscr{M}$. 
iv) The final step in our construction is the reduction to the quotient manifold $\mathscr{M}$ of the Poisson tensors (4.2) and (4.3) carried by the loop superalgebra. For simplicity, the reduced tensors are indicated again by $Q$ and $P$; according to [CMP , CP], they are defined as follows at any point $m$ of the quotient space:

$$
Q_{m} \delta m=\left(T_{S} \pi\right)\left(Q_{S} \delta V\right), \quad P_{m} \delta m=\left(T_{S} \pi\right)\left(P_{S} \delta V\right) .
$$

Here, $S$ is any point of the fiber $\pi^{-1}(m)$ and $T_{S} \pi: T_{S} \mathscr{S} \rightarrow T_{m} \mathscr{M}$ is the tangent map to the projection; $\delta V \in \mathscr{G}_{0}$ is any covector which annihilates the distribution $\mathscr{D}_{S}$ and projects on $\delta m$ in the following sense: $\langle\delta V, \dot{S}\rangle=\left\langle\delta m,\left(T_{S} \pi\right) \dot{S}\right\rangle$ for each $\dot{S} \in T_{S} \mathscr{S}$.

Applying Eq. (4.11), we find for the Poisson tensors $Q_{m}$ and $P_{m}$ the explicit expressions given in Table 1a of Sect. 2. In conclusion, we have obtained a biHamiltonian structure on the quotient space $\mathscr{M}$; now, if we look for the biHamiltonian hierarchies engendered by this structure, we are led to the susy AKNS theory of Sect. 2.

\section{Description of the Method: The Lax Formalism}

In the biHamiltonian strategy, the Lax formalism is a supplementary outcome of the reduction of loop algebras; the general procedure was discussed in [MP2], and here we will apply it to the susy AKNS theory.

Consider the loop superalgebra $\mathscr{G}$ introduced in the previous section. The elements of $\mathscr{G}$ are $4 \times 4$ matrices where each entry is a superfield; so, they act naturally by multiplication on the quadruples of superfields $\left(\begin{array}{l}\Psi_{1}(x, \theta) \\ \Psi_{2}(x, \theta) \\ \xi_{1}(x, \theta) \\ \xi_{2}(x, \theta)\end{array}\right)$. Following

Subsect. 2.2, let us introduce a space $\mathscr{W}$ of such quadruples, and a space $\operatorname{Lin}^{\lambda}(\mathscr{W})$ of linear operators on $\mathscr{W}$, depending polynomially on the parameter $\lambda$. Furthermore, let us fix attention on the section $\Sigma: \mathscr{M} \rightarrow \mathscr{S} \subset \mathscr{G}_{1}$ defined by Eq. (4.10), and introduce the map

$$
\mathscr{L}^{\lambda}: \mathscr{M} \rightarrow \operatorname{Lin}^{\lambda}(\mathscr{W}), \quad m \mapsto \mathscr{L}^{\lambda}(m):=D+\Sigma(m)+\lambda A
$$

( $A$ is the matrix in Eq. (4.2)). The biHamiltonian reduction scheme from which the map $\Sigma$ arises implies that $\mathscr{L}^{\lambda}(m)$ is a matrix Lax operator for the susy AKNS hierarchy. The explicit expression of this operator was anticipated in Sect. 2 (see Table 1b).

We now explain how to construct maps $\mathscr{C}_{j}^{\lambda}: \mathscr{M} \rightarrow \operatorname{Lin}^{\lambda}(\mathscr{W}), m \mapsto \mathscr{C}_{j}^{\lambda}(m)$ such that the Lax formulation $d \mathscr{L}^{\lambda}(m) / d t_{j}=\left[\mathscr{L}^{\lambda}(m), \mathscr{C}_{j}^{\lambda}(m)\right]$ holds. First of all, one checks by direct computation that this equation is satisfied for $j=-1$, setting $\mathscr{C}_{-1}^{\lambda}(m)=\mathscr{Z}$, with $\mathscr{Z}:=\operatorname{Diag}(-1,1,-1,1)$. The biHamiltonian reduction theory ensures that all the other vector fields of the hierarchy admit a Lax representation and also allows the following geometrical construction for the operators $\mathscr{C}_{j}$.

Let $m \in \mathscr{M}, \delta m \in T_{m}^{*} \mathscr{M}, S=\Sigma(m)$. Then there exist two covectors $\delta V, \delta V^{\prime} \in$ $T_{S}^{*} \mathscr{G}_{1} \approx \mathscr{G}_{0}$, such that the linear combination $\delta V^{\lambda}=\delta V+\lambda \delta V^{\prime}$ has the following properties, identically in $\lambda$ :

i) $\delta V^{\lambda}$ annihilates $\mathscr{D}_{S}$;

ii) $\delta V^{\lambda}$ projects on $\delta m$, i.e., $\left\langle\delta V^{\lambda}, \dot{S}\right\rangle=\left\langle\delta m,\left(T_{S} \pi\right) \dot{S}\right\rangle$ for each $\dot{S} \in T_{S} \mathscr{S}$;

iii) $\left(P_{S}+\lambda Q_{S}\right) \delta V^{\lambda} \in \operatorname{Im}\left(T_{m} \Sigma\right)$ (i.e., this vector is tangent to the cross section $\Sigma$ ). 
The $\lambda$-dependent covector $\delta V^{\lambda}$ is uniquely determined by the above conditions; putting

$$
\Sigma^{\star \lambda}(m, \delta m):=\delta V^{\lambda}
$$

we define a map $\Sigma^{\star \lambda}$ from $T^{*} \mathscr{M}$ to $T^{*} \mathscr{G}_{1}[\lambda]$ (the bundle whose fiber at a point $V$ is the space $T_{V}^{*} \mathscr{G}_{1}[\lambda] \approx \mathscr{G}_{0}[\lambda]$, formed by the covectors depending polynomially on the parameter $\lambda$ ). The explicit expression of this map is reported in Table 1b; according to the terminology introduced in [MP2], $\Sigma^{\star \lambda}$ is the ( $\lambda$-dependent) lift map corresponding to the cross section $\Sigma$. Let us now put $\mathscr{C}_{j}^{\lambda}(m)=\sum_{k=0}^{j}(-1)^{k} \lambda^{k} \Sigma^{\star \lambda}\left(m, d_{j-k}(m)\right)+(-1)^{j+1} \lambda^{j+1} \mathscr{Z}$, where $d_{j}$ denotes, as before, the differential of the $j^{\text {th }}$ Hamiltonian function. The maps $\mathscr{C}_{j}^{\lambda}$, together with $\mathscr{L}^{\lambda}$, give the Lax representation of the hierarchy .

This representation is realized in terms of $4 \times 4$ matrix superdifferential operators; we now infer some "reduced" Lax formulations in terms of $2 \times 2$ matrix operators, applying a reduction theory for parameter-dependent Lax pairs discussed in [MP2]. Let us examine the spectral problem $\mathscr{L}^{\lambda}(m) \Phi^{\lambda}=0$; this problem was considered in Sect. 2, where we found that it is equivalent to the linear system (2.2.6).

We now reformulate this fact in a more geometrical fashion. The Lax operator $\mathscr{L}^{\lambda}(m)$ acts on a space $\mathscr{W}$ of quadruples $\Phi=\left(\begin{array}{c}\Psi_{1} \\ \Psi_{2} \\ \xi_{1} \\ \xi_{2}\end{array}\right)$, where each component is a superfield. Let us identify $\mathscr{W}$ with a direct sum:

$$
\mathscr{W}=\mathscr{H} \oplus \mathscr{H}^{\prime},
$$

where the space $\mathscr{H}$ consists of pairs $\Psi:=\left(\begin{array}{l}\Psi_{1} \\ \Psi_{2}\end{array}\right)$ and $\mathscr{H}^{\prime}$ consists of pairs $\xi:=\left(\begin{array}{l}\xi_{1} \\ \xi_{2}\end{array}\right)$. Due to this decomposition, we can represent a linear operator from $\mathscr{W}$ into itself as a matrix $\left(\begin{array}{ll}T & X \\ Y & Z\end{array}\right)$, where $T$ maps $\mathscr{H}$ into itself, $X$ sends $\mathscr{H}^{\prime}$ into $\mathscr{H}$, etc.. The linear system (2.2.6) can be written as

$$
\left(\begin{array}{cc}
-L(m)+\lambda & 0 \\
-R(m) & 1
\end{array}\right)\left(\begin{array}{c}
\Psi \\
\xi
\end{array}\right)=0
$$

with $L(m)$ as in Table $1 \mathrm{~b}$ and $R(m) \Psi:=\left(\begin{array}{c}\Psi_{1}^{\prime}-\frac{1}{2} \psi \Psi_{2} \\ \Psi_{2}^{\prime}-\frac{1}{2} \varphi \Psi_{1}\end{array}\right)$.

Note that the matrix appearing in Eq. (5.4) is lower triangular. In fact, the operator $\mathscr{L}^{\lambda}(m)$ admits a triangular decomposition, in the sense of [MP2]; this implies that the Lax formulation (2.2.5) can be reduced to a parameter independent formulation of the form (2.2.8), in terms of linear operators acting on $\mathscr{H}$. To this purpose, we define two linear maps

$$
\begin{aligned}
& E: \mathscr{W} \rightarrow \mathscr{H}, \quad E\left(\begin{array}{c}
\Psi \\
\xi
\end{array}\right):=\Psi, \\
& \mathscr{F}(m): \mathscr{H} \rightarrow \mathscr{W}, \quad \mathscr{F}(m) \Psi:=\left(\begin{array}{c}
\Psi \\
R(m) \Psi
\end{array}\right) .
\end{aligned}
$$

Let us write down each of the operators $\mathscr{C}_{j}^{\lambda}(m) \in \operatorname{Lin}^{\lambda}(\mathscr{W})$ as a polynomial in $\lambda: \mathscr{C}_{j}^{\lambda}(m)=\sum_{k=0}^{j+1} \mathscr{C}_{j k}(m) \lambda^{k}$, and define as follows the operators $C_{j}(m)$, acting 
on $\mathscr{H}$ :

$$
C_{j}(m):=\sum_{k=0}^{j+1} E \mathscr{C}_{j k}(m) \mathscr{F}(m) L^{k}(m)
$$

(i.e., multiply $\mathscr{C}_{j}^{\lambda}(m)$ by $E$ on the left, $\mathscr{F}(m)$ on the right and replace $\lambda^{k}$ with the $k^{\text {th }}$ power of $\left.L(m)\right)$. We can employ $L(m)$ and the above operators to set up a Lax formulation as in Eq. (2.2.8); also, from the definitions of $\mathscr{C}_{j}^{\lambda}(m), E, \mathscr{F}(m), R(m)$, it follows that the operators $C_{j}(m)$ can be expressed as in Table $1 \mathrm{~b}$.

Acknowledgements. We are grateful to $\mathrm{P}$. Kersten for bibliographical indications. This paper has been partially supported by Consiglio Nazionale delle Ricerche, G.N.F.M, and by Ministero dell'Università e della Ricerca Scientifica, Project "Metodi Geometrici e Probabilistici in Teorie di Campo."

\section{References}

[CMP] Casati, P., Magri, F., Pedroni, M.: Bihamiltonian manifolds and the $\tau$-function. Proceedings of the 1991 Joint Summer Research Conference on Mathematical aspects of Classical Field Theory. M. Gotai, J. Marsden, V. Moncrief (eds.) in Contemp. Math. 132, 213-234 (1992)

[CN] Chowdhury, R.A., Naskar, M.: On the complete integrability of the supersymmetric nonlinear Schrödinger equation. J. Math. Phys. 28, 1809-1812 (1987)

[Cor] Cornwell, J.: Group theory in Physics. Vol. III. London: Academic Press (1989)

[CP] Casati, P., Pedroni, M.: Drinfeld-Sokolov reduction on a simple Lie algebra from the biHamiltonian point of view. Lett. Math. Phys. 25, 89-101 (1992)

[DS] Drinfeld, V., Sokolov, V.: Lie algebras and equations of Korteweg-de Vries type. J. Sov. Mat. 30, 1995-2036 (1985)

[EW] Estabrook, F.B., Wahlquist, W.D.: Prolongation structures of nonlinear evolution equations II. J. Math. Phys. 17, 1293-1297 (1976)

[FF] Fuchssteiner, B., Fokas, A.S.: Symplectic structures, their Bäcklund transformations and hereditary symmetries. Physica D 4, 47-66 (1981)

[FMR] Figueroa-O'Farrill, J., Mas, J., Ramos, E.: Integrability and biHamiltonian structure of the even order sKdV hierarchies. Rev. Mat. Phys. 3, 479-501 (1991)

[IK1] Inami, T., Kanno, H.: Lie superalgebraic approach to super Toda lattice and generalized super KdV equations. Commun. Math. Phys. 136, 519-542 (1991)

[IK2] Inami, T., Kanno, $\mathrm{H} .: N=2$ super $\mathrm{KdV}$ and super-sine Gordon equations based on Lie superalgebra $A(1,1)^{(1)}$. Nucl. Phys. B 359, 201-217 (1991)

[IK3] Inami, T., Kanno, H.: $N=2$ super- $W$-algebras and generalized $N=2$ super $\mathrm{KdV}$ hierarchies based on Lie superalgebras. J. Phys. A 25, 3729-3736 (1992)

[IK4] Inami, T., Kanno, H.: Generalized $N=2$ super KdV hierarchies: Lie superalgebraic methods and scalar super Lax formalism. Proceedings of the RIMS Research Project 1991, "Infinite Analysis," in Int. J. Mod. Phys. A 7, (Suppl. 1 A) 419-447 (1992)

[Kul] Kulish, P.P.: ICTP, Trieste preprint IC/85/39 (1985)

[Kup] Kupershmidt, B.: A super Korteweg-de Vries equation. Phys. Lett. 102, A 213-215 (1984)

[Lei] Leites, D.: Introduction to the theory of supermanifolds. Russ. Math. Surv. 35, 1-64 (1980)

[LM] Laberge, C., Mathieu, P.: $N=2$ superconformal algebra and integrable $O(2)$ fermionic extension of the KdV equations. Phys. Lett. 215 B, 718-722 (1988)

[LiM] Liberman, P., Marle, C.M.: Symplectic geometry and analytical mechanics. Dordrecht: Reidel, (1987)

[Ma1] Magri, F.: A simple model of the integrable Hamiltonian equation. J. Math. Phys. 19, 1156-1162 (1978) 
[Ma2] Magri, F.: A geometrical approach to the nonlinear evolution equations. Proceedings of the 1979 Lecce Meeting on "Nonlinear evolution equations and dynamical systems", M. Boiti, F. Pempinelli, G. Soliani (eds.) Lect. Notes in Phys. 120, New York: Springer, pp. 233-263, (1980)

[MaR] Marsden, J.E., Ratiu. T.: Reduction of Poisson Manifolds. Lett. Math. Phys. 11, 161-169 (1986)

[Mat] Mathieu, P.: Supersymmetric extension of the Korteweg-de Vries equation. J. Math. Phys. 29, 2499-2506 (1988)

[MMR] Magri, F., Morosi, C., Ragnisco, O.: Reduction techniques for infinite-dimensional Hamiltonian systems: Some ideas and applications. Commun. Math. Phys. 99, 115-140 (1985)

[MP1] Morosi, C., Pizzocchero, L.: On the biHamiltonian structure of the supersymmetric KdV hierarchies: A Lie superalgebraic approach. Commun. Math. Phys. 158, 267-288 (1993)

[MP2] Morosi, C., Pizzocchero, L.: On the biHamiltonian interpretation of the Lax formalism. Rev. Math. Phys. 7, 389-430 (1995)

[MP3] Morosi, C., Pizzocchero, L.: Osp $(3,2)$ and gl $(3,3)$ supersymmetric KdV hierarchies. Phys. Lett. A 185, 241-252 (1994)

[MP4] Morosi, C., Pizzocchero, L.: On the equivalence of two supersymmetric KdV theories: A biHamiltonian viewpoint. J. Math. Phys. 35, 2397-2407 (1994)

[MR] Manin, Y., Radul, A.: A supersymmetric extension of the Kadomtsev-Petviashvili hierarchy. Commun. Math. Phys. 98, 65-77 (1985)

[OP] Oevel, W., Popowicz, Z.: The biHamiltonian structure of fully supersymmetric Kortewegde Vries systems. Commun. Math. Phys. 139, 441-460 (1991)

[Pop] Popowicz, Z.: The extended supersymmetrization of the Nonlinear Schrödinger equation. Preprint 9/6/1994, Univ. of Wroclaw

[RH] Roelofs, G.H.M., van den Hijligenberg, N.W.: Prolongation structures for supersymmetric equations. J. Phys. A. Math. Gen. 23, 5117-5130 (1990)

[RK] Roelofs, G.H.M., Kersten, P.H.M.: Supersymmetric extensions of the nonlinear Schrödinger equation: symmetries and coverings. J. Math. Phys. 33, 2185-2206 (1992)

Communicated by R.H. Dijkgraaf 
